TRANSIEPT COMPRESSIBLE FLOWS IN POROUS MEDIA

Frank A. Morrison, Jr.

Department of Mechanical and Industrial Engineering

University of Illinois at Urbana-Champaign

Urbana, IL 61801

Final Report (UILU ENG 75 4004)

of Research Performed under

Subcontract Ho. 1160305

Tor

THE REGENTS OF THE UNIVERSITY OF CALIFORNIA

September 1975

Copy No. 2 of Ten (10) Copies

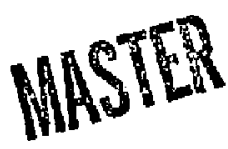




\section{TRANSIENT COMPRESSIBLE FLOWS IN POROUS MEDIA}

Frank A. Marrison, Jr. Department of Mcchanical and Industrial Engineering University of Illinois at Urbana-Champaign Urbana, IL 61801

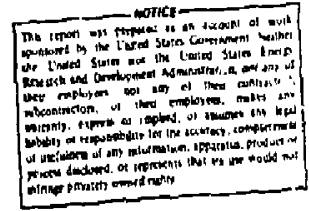

Final Report (UILU ENG 75 4004)

of Research Perionted under

Subcontract No. 1160305

for

THE REGENTS OF THE UHIVERSITY OF CALIFORUIA

Septeniber 1975 
Now

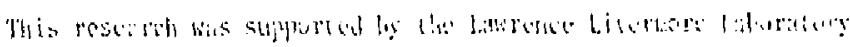

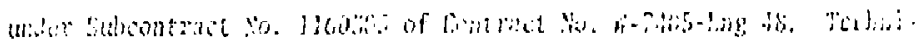

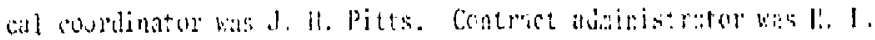
Ha1kikN.

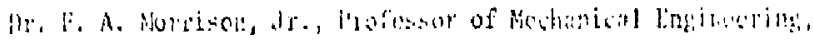

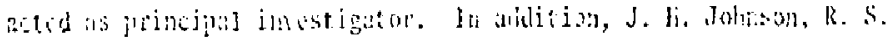

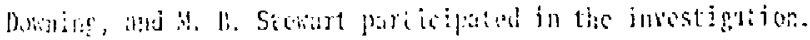


Aivilu:

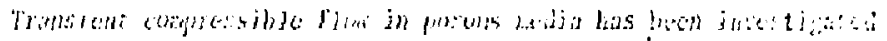

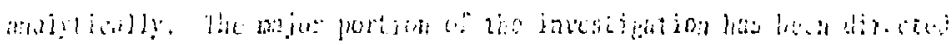

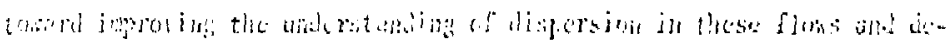

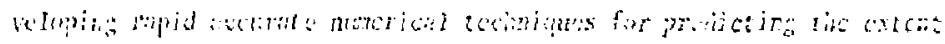

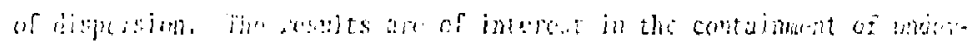

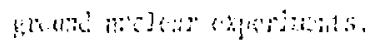

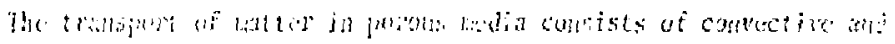

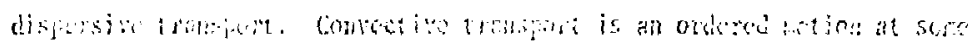

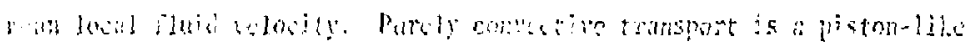

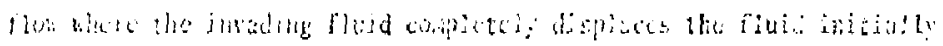

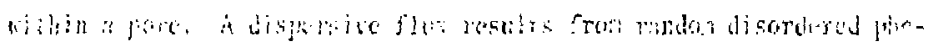

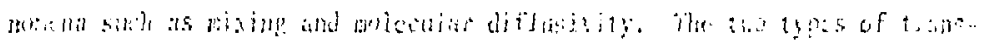

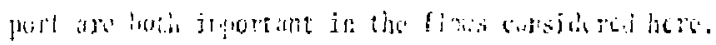

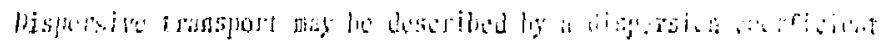

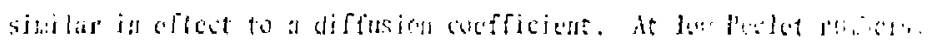

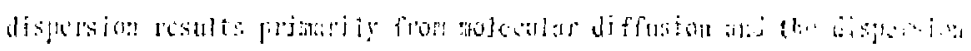

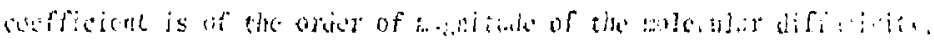

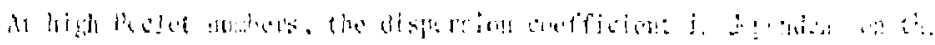

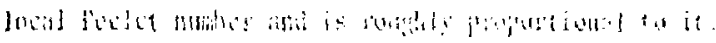

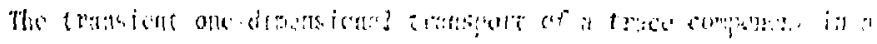

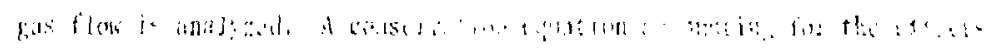

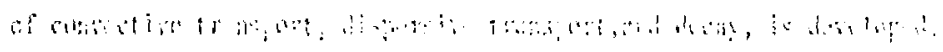

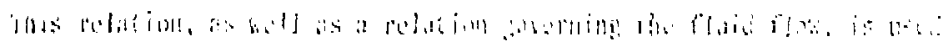

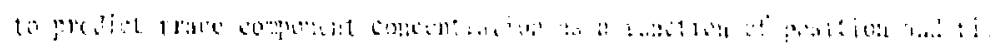

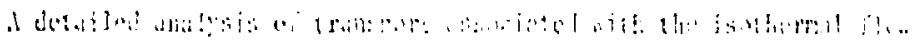

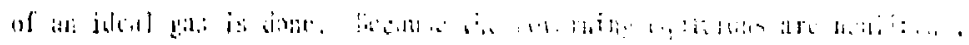




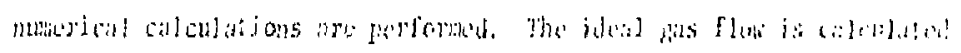

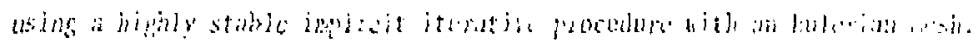

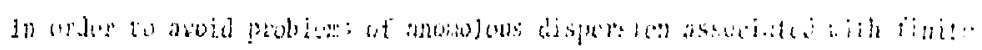

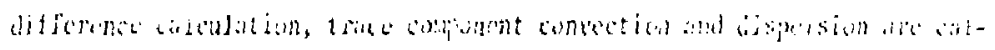

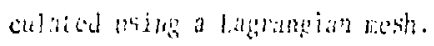

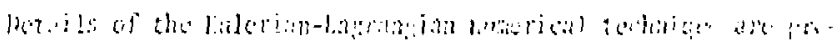

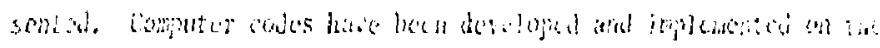

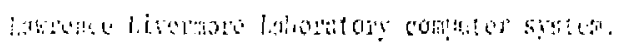


TABII: (W: COKTE...TS

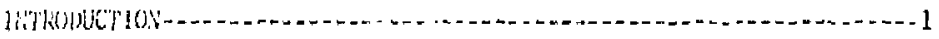

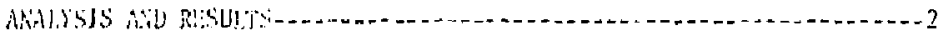

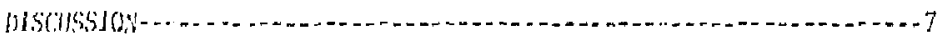

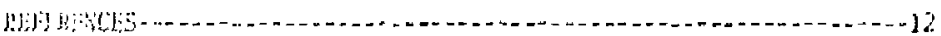

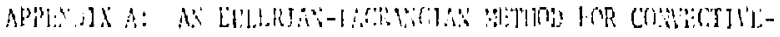

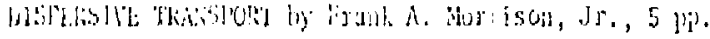

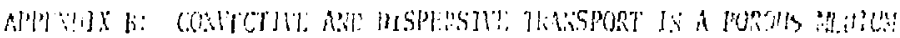

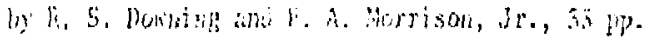

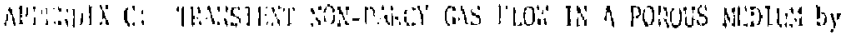

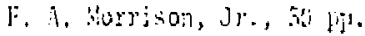




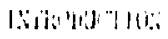

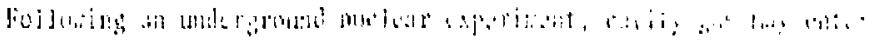

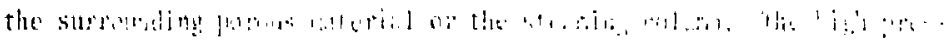

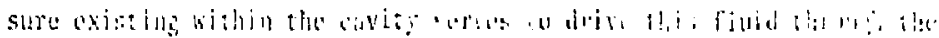

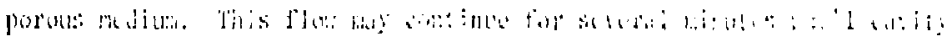

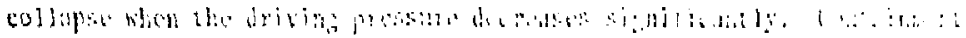

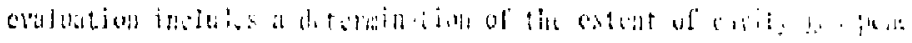
tration.

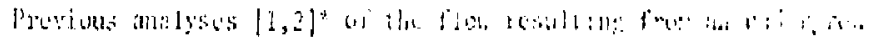

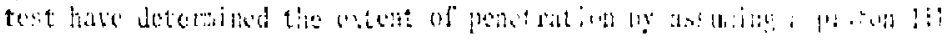

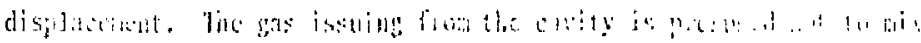

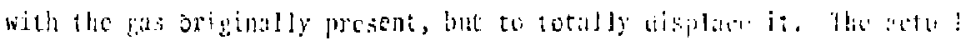

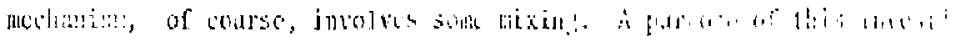

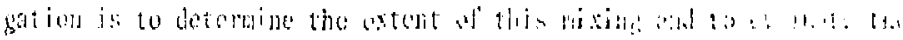
utilizy of the piston fuor anduximatin. 


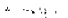

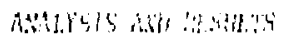

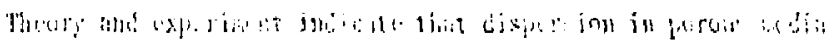

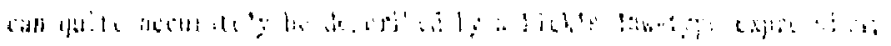

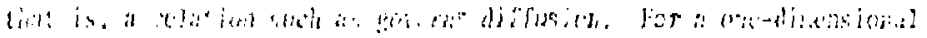

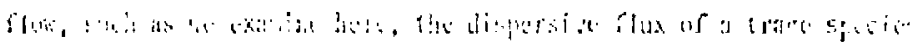
is $14+1616 i$

$$
n_{1}=-11
$$

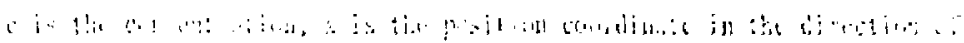

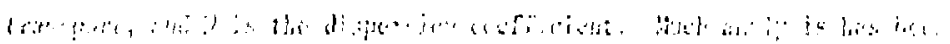

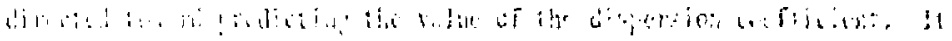

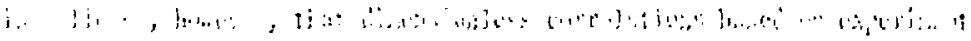

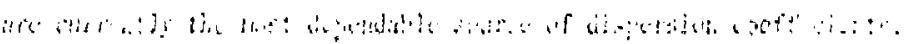

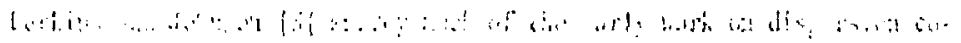

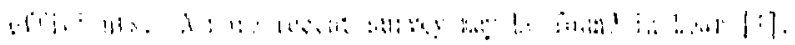

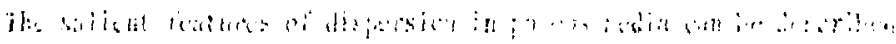

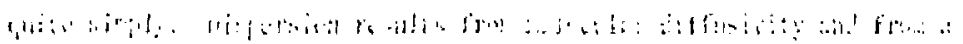

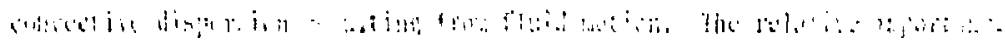

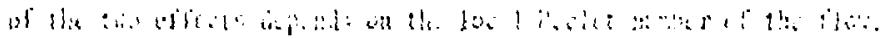

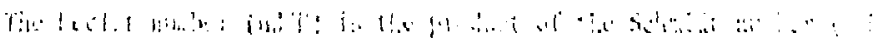

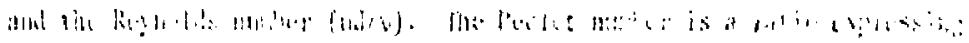

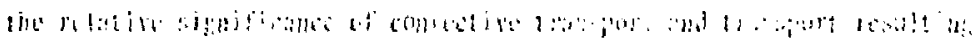

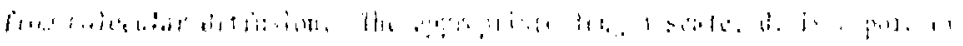

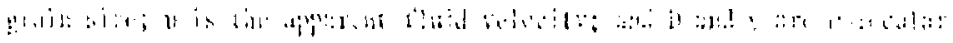

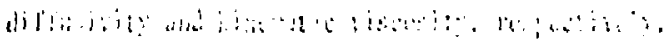

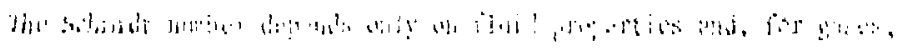

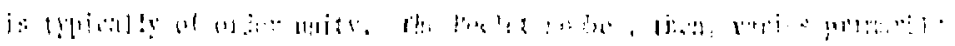

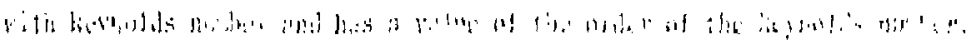

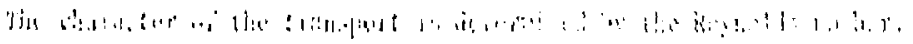




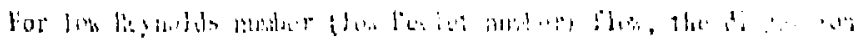

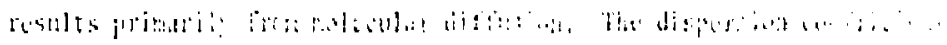

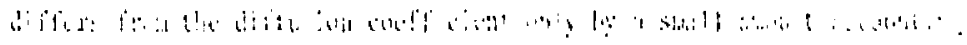

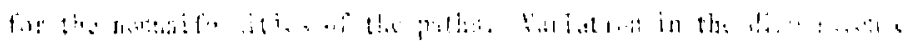

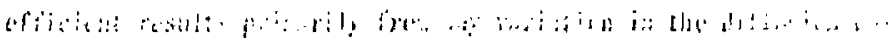

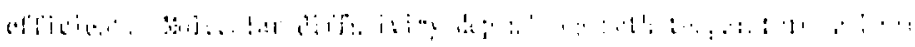
shese 11 .

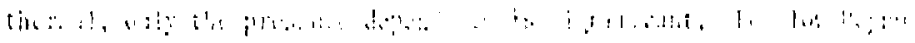

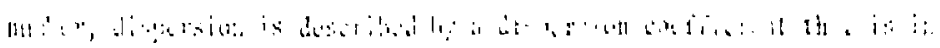

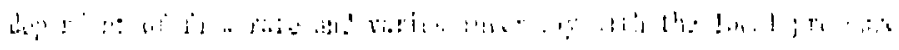

$$
u=v_{0} \frac{i}{1}
$$

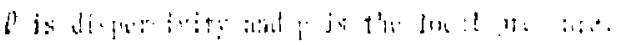

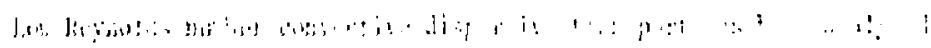

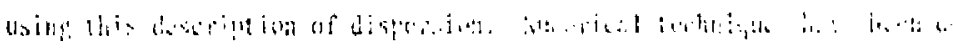

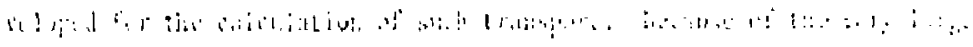

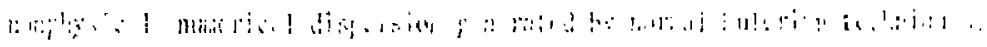

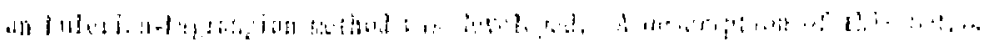

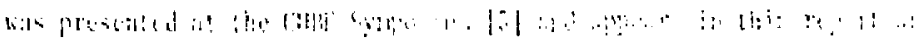
:1iti in 111

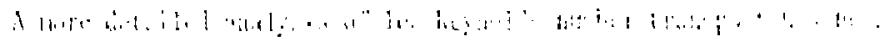

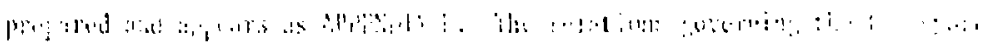

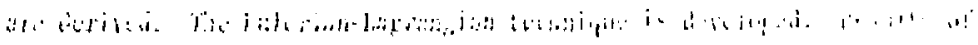

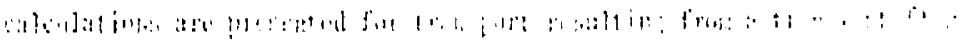

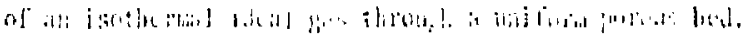

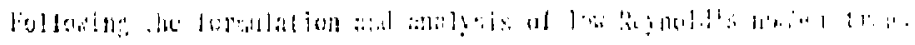

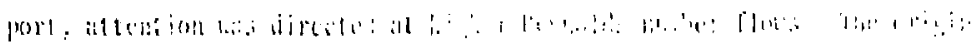




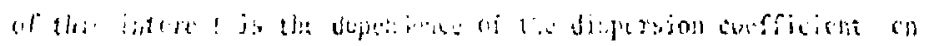

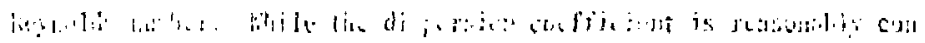

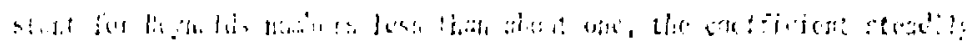

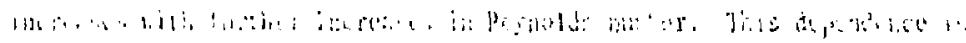
i! (x) -

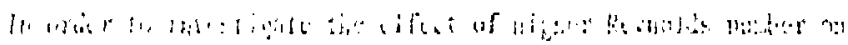

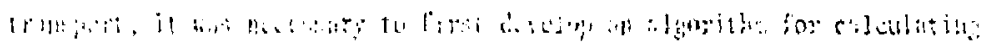

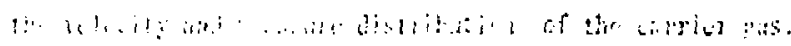

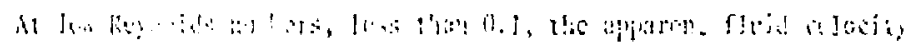

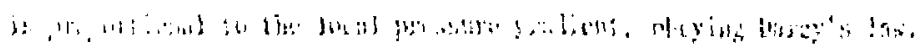

$$
u=\frac{3 .}{i} \text { in }
$$

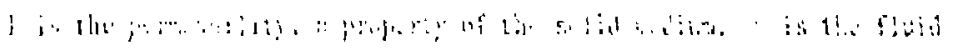

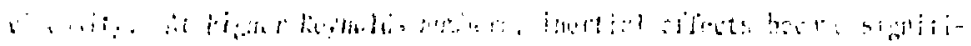

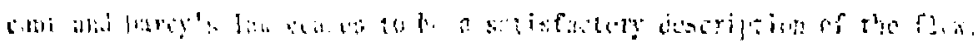

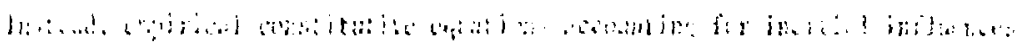

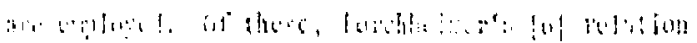

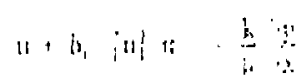

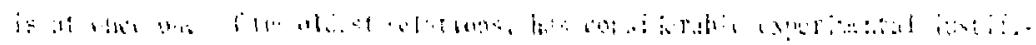

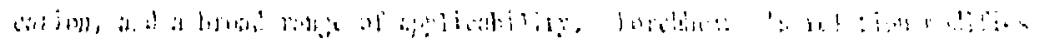

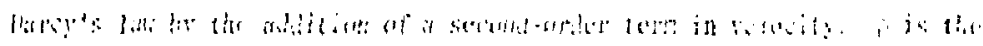

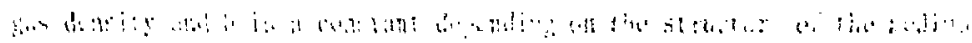

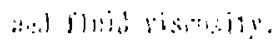

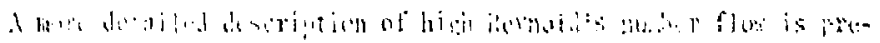

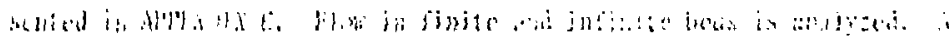

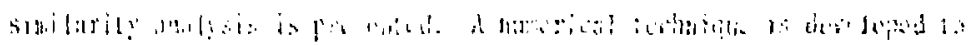

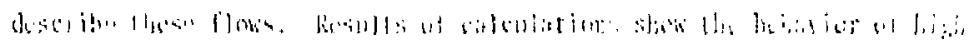




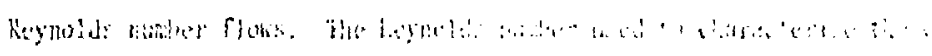
Hons: is

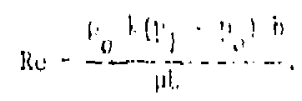

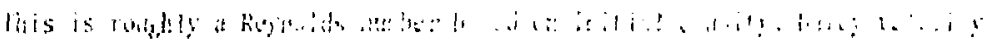

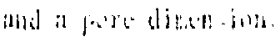

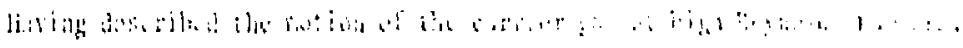

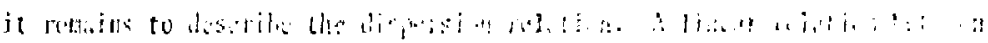

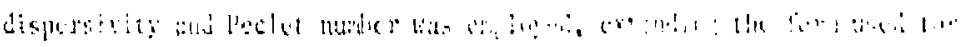

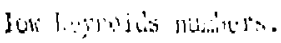

$$
v=D_{0} \underset{0}{g_{0}}(1+1+\cdots \cdots
$$

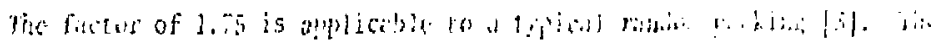

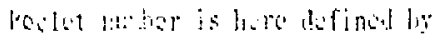

$$
p_{c}=\frac{u l}{p_{0} p_{0} / p}
$$

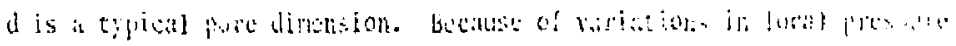

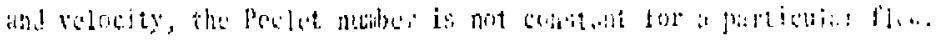

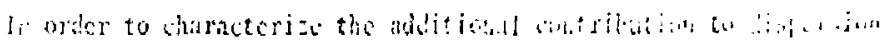

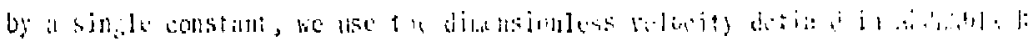

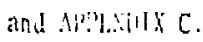

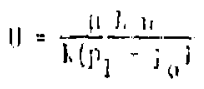

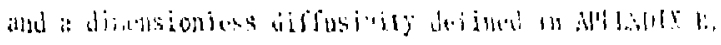

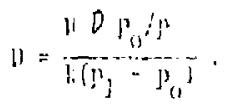

In terns of these variables, his have

$$
P C=\frac{11}{11} \frac{1}{1} \text {. }
$$




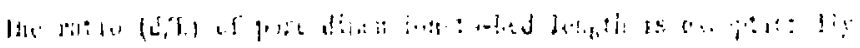

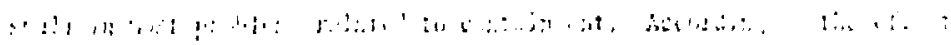

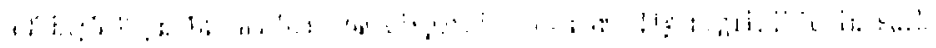
$+\ldots, \ldots, \ldots$

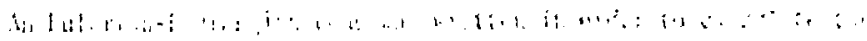

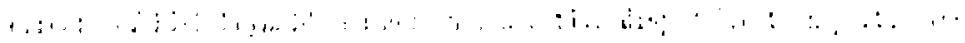

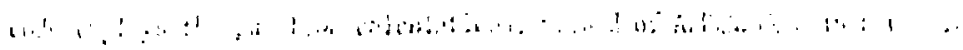

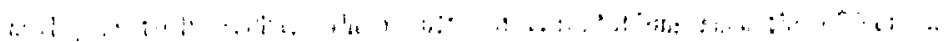

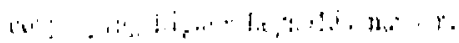

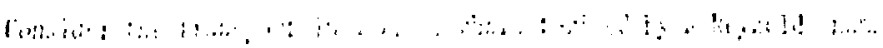

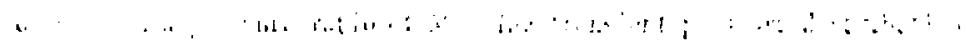

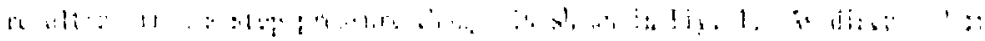

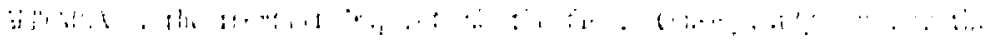

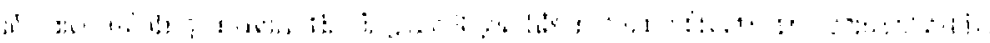

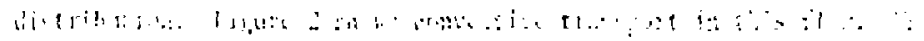

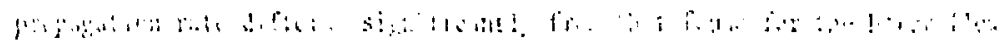

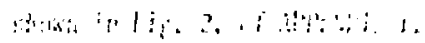

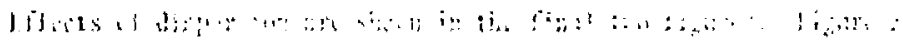

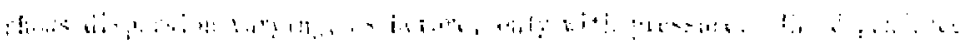

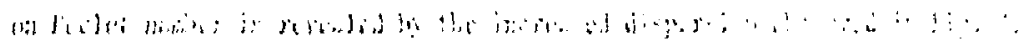

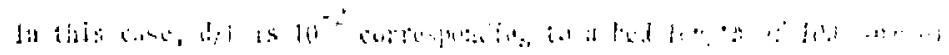

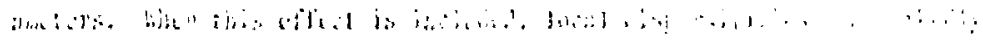

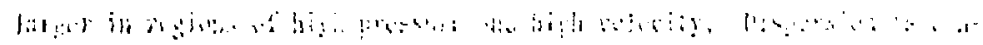

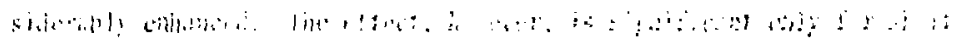

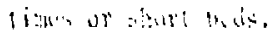




\section{DISCUSSion}

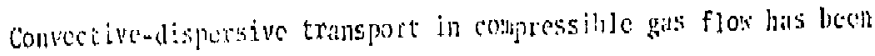

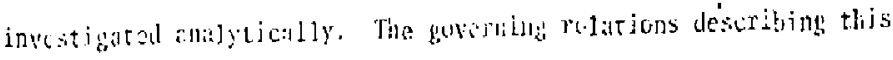

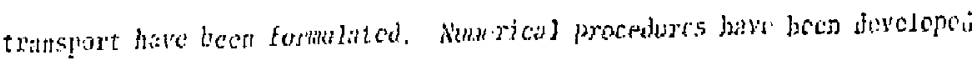

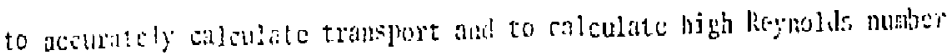
gis floti. kosulte of such salculations hime been prosented and discussid. 


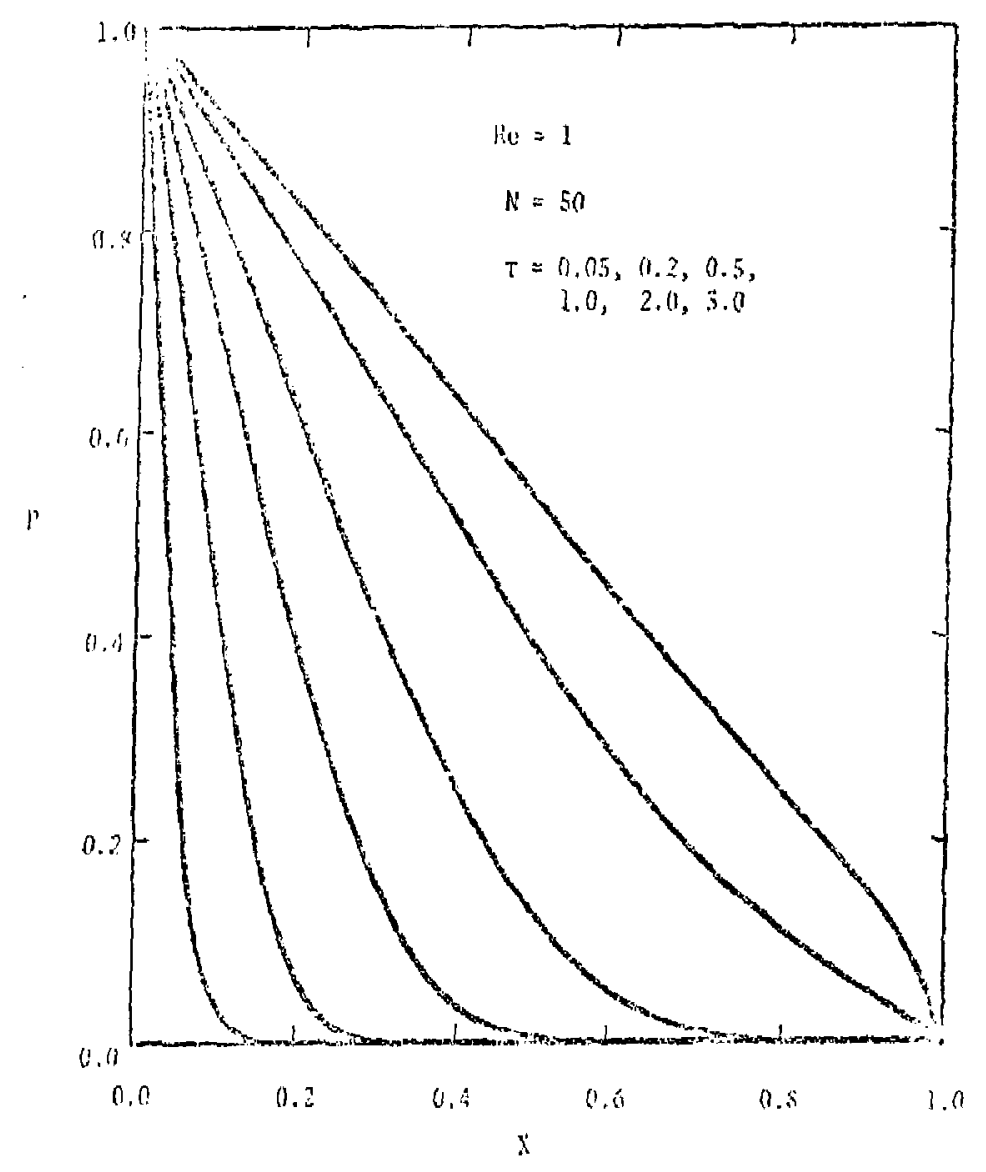

Fare 


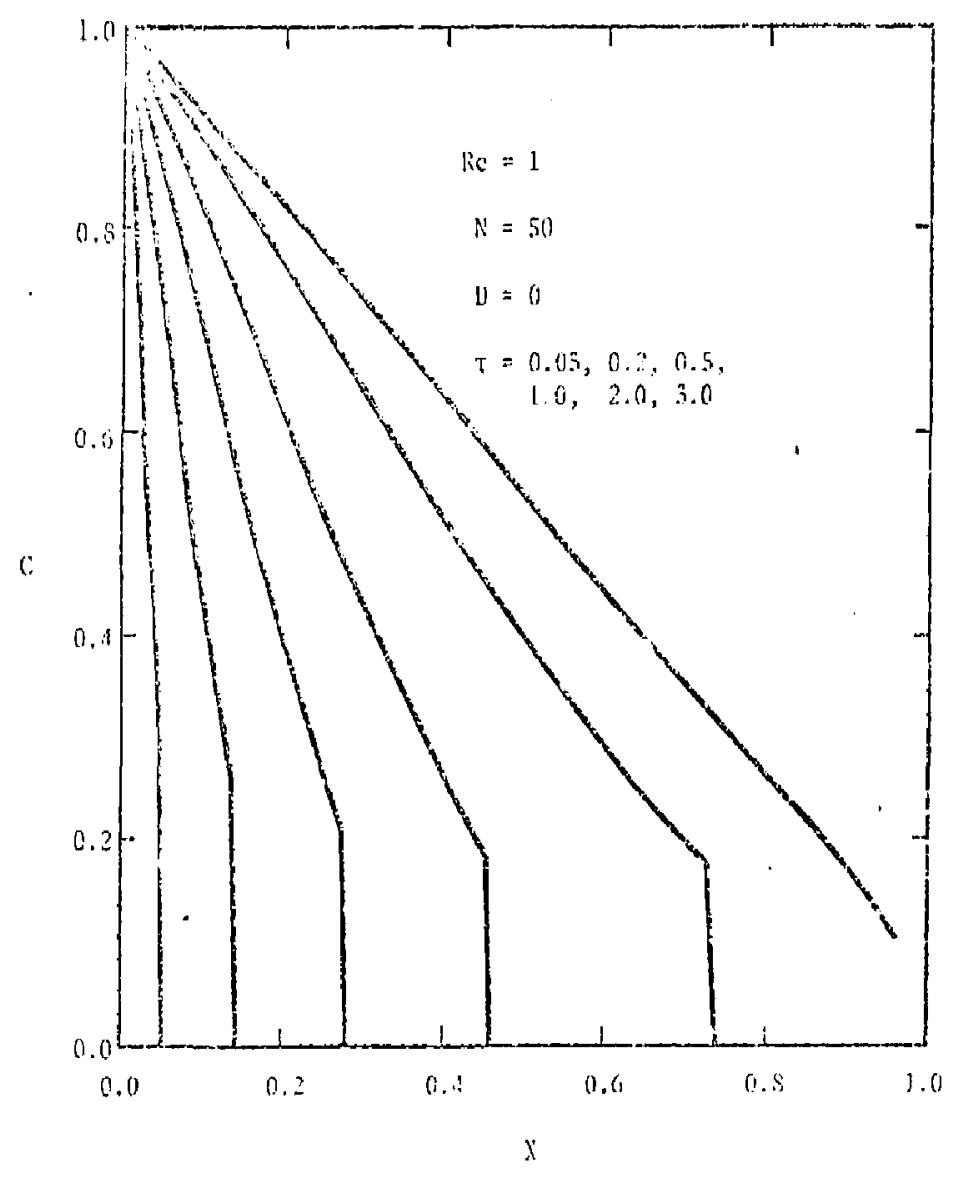

byan? 


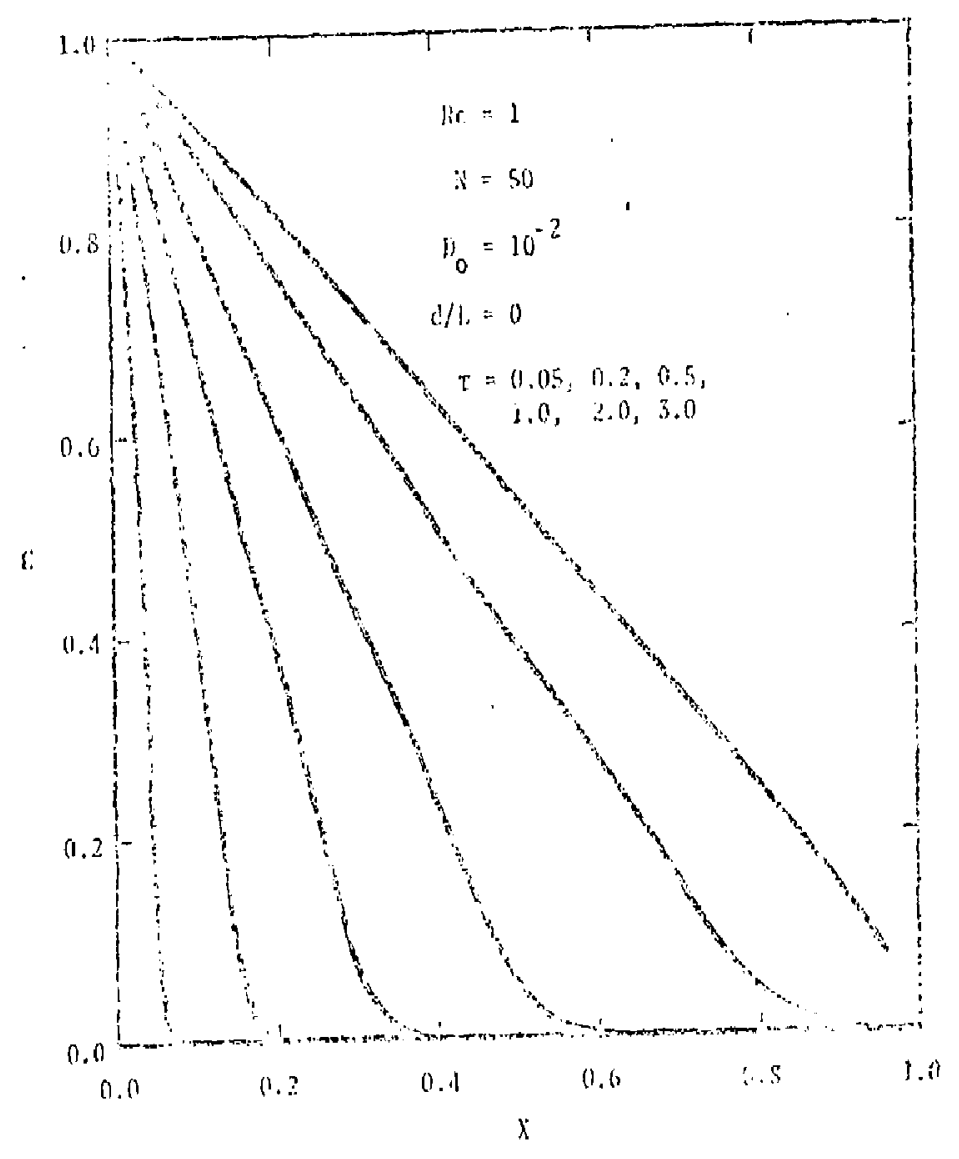

jitire 5 


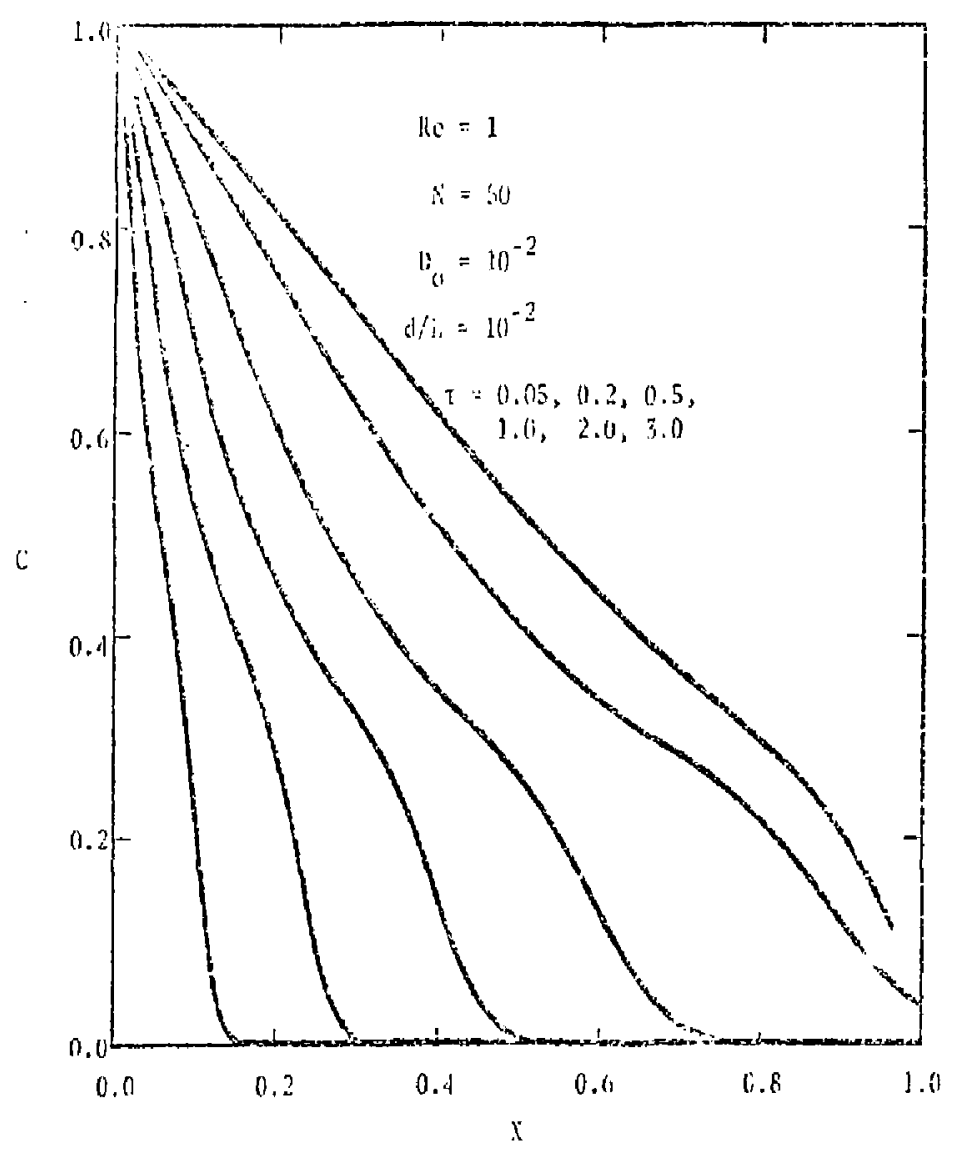

Ii:ut: 


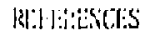

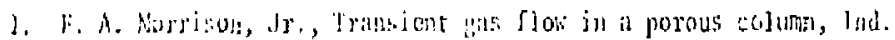
Eng. (helin, fund, 1], 191 (197.).

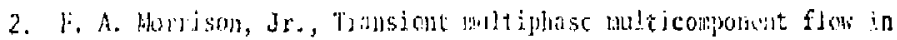

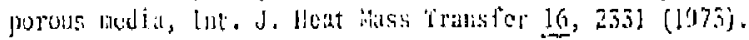

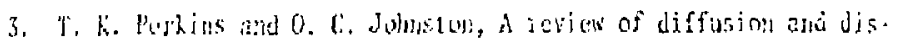

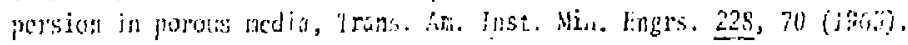

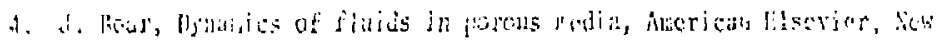
riask (197) .

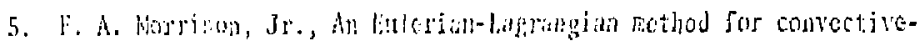

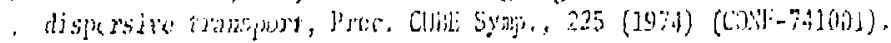

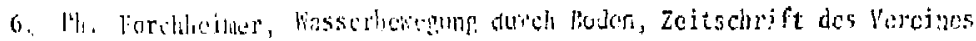

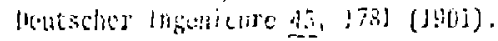




\section{APPENDIX A}

AN EULERIAN-LAGRANGIAN METHOD FOR

CONVECTIVE-DISPERSIVE TRANSPORT

Frank A. Morrison, Jr. 


\author{
A. DULERIAK-LAGRAMGIAN BETHOD YOR \\ CONYECTIVE-DISPERSIVE TRUNSFORT \\ Frink A, Morpiston, in. \\ Lawence Livemore Laboratory \\ Professor of thengical Engineering \\ Latversity of Illinois at Urbana-Champaign \\ Unband, II. 61801
}

\title{
ABstact
}

Convective-dispersive transport in a one-dimensional compressible flow is solved using an Eulorian-Lagrangian technique. The transport of a tagged species, resulting from dispersion and ges flok in porous medir, is studied. The velocity distribution of the bulk gas totion is caleulated using at Eulerian vievpoint. This velocity dis= tribution detemines the motion of the Latrangian asth. The silxed Eulerian-Lagrangian approach is adopted as a means of avoiding anomolous numerical dispersion. A Lagrangian wh noves along charaterist ic curves of the convective transport equation. Any transport relative to this mesh is dispersive. In the absence of physical dispersior, there is no transfer of the species between Lagraugian mesh points and consestration shanges then result solely from compression and exparsion of the fluid element. Because the flow is compressible, th: Lagrangian mesh points are unequally spaced. Inplicit methods for solution of the diffusion equation are dereloped for unequally spaced mesh points. Retailed calculations have bten done for the transient, low Reynolds number, footherral flow of an ideal gas.

\section{IKTROBUCTION}

Convective and dispersive transport are associated with many processes of engineering significanct. Techriques developed for the analysis of the relations governing these phenomena may, conse Jusently, have wide applicability. Here, wa present some techniques developed in the analysis of tiscible displacement in a transient compressible flow tirrough porous media. Because the relation governing ile bulk fluid velccity distribution is nonlinear, numeri eal techniques are used. We examine difficulties encountered in the numerical som lution of the convective-dispersive transport equation and describe means of overconing these difficulties.

Ne consider the transport in one space dimension of a trace component charseterized by a concentration $c$. $c$ is a function tf position $x$ and time $t$. The concentrution digtribution is to be detemined. The poverning relition is otrtalned simply by applyine conservation of epecies to an infinitesimal elentent of space. The convect ive flux deross a unit control surface normal to the direction of flor is uc. $u$ is the apparent ve* loelty, volume flay rate per unit normal area, of the currier gas, The Jispersive transport is nomdlly considered, tithe diffusion, to obcy a reistion of the form of Rick's law. Using a dispersion coeffleient, $D$, the $f l u x$ is $-D(\Delta c / \partial x)$. Equating the net flux into the element to the rate of accurala jon within the eltient, she transport equation results.

$$
c \frac{\partial c}{\partial t}+\frac{\partial}{\partial x}\left(w c-0 \frac{\partial c}{\partial x}\right)=0
$$

$\varepsilon$ is the porosity of the $\pi^{2} \mathrm{~m}^{2} \mathrm{iut}$, the roid valune fraction.

In an incompressible flow in the dimension, the velocity $y$ is constant. Additionally, the dispersivity is often taken tc be constant. Heicher simglification is valid in our application. The equation governing the concentrat ten distribution has nonconst unt cowifizionts. $y$ and $D_{*}$ functioni of position and the and delerminud in numuriesl tedini fues. The velacley and dituperivivity rin'ule iron solution of the eyuation yovernitit: tulk gas wction.

\section{woton or tit: rimin}

the fore in mich the rivis velocity distribut ion hecome: hnow "ffirect: the subsequent choice of tooli utilizint ints distributisn.

The law Reynolds; number fluw of an ispthemal idral gat in une dirpusion 
obeys a well knomm ${ }^{1,2}$ relation

$$
\frac{\partial}{\partial x}\left(p \frac{\partial p}{\partial x}\right)=\frac{c \mu}{k} \frac{\partial p}{\partial t}
$$

P is the local gas pressure, while $\checkmark$ is the gas viscosity and $k$, the pertoeaility of the mediun. The gas velocity 13. expressed in cerns of the pressure gudfent by Darcy's law.

$$
u=-\frac{k}{\mu} \frac{\partial p}{\partial x}
$$

The dispersion coefficient is independent of velocity in the low Raynolds number, low Peclet number range. It does, however, rary inversely with the gas pressure

$$
D=D_{0} \frac{P_{0}}{p}
$$

The subscript o refers to ambient conditions, Calculation of the pressure distribution yields the velocity and dispersivity dtstributions.

Equation (2) is analogous tc a nonlinear heat conduction equation. The nuancical procedure shosen for lts salution 14 the method of Bruce, Peaceman, Ract:ford, and RIce.' This is an implicit, unconditionally stable procedure similar to the Crank-Nlcolson" method for the linedr diffusion equation. Because of the nonLinearlty of Eq. (2), the procedure is Iteratlve. However, the converence is rapld, Horeover, the coefficient matrix of the finite difference equations is tridiogonal so that each iteration is efficiently perforned using Thomas" altorl then.

\section{MHERICAL DISPERSLOH}

Becaude an tulerian approuch is used to doternine the velocity jol dispersivity distribut lons, Lulerian mithods sljould be considured for fuberequent unulysis of the trace coaponcht transport. Eulerlin techniques, liourver, tend to profluce an anowolass nunoricsl ditipurtion which could eacily exced the pliysical disperaton of interest.

Considor, as on axapple, convect ive trunsport in an incorpinitsililo flow. In this ulmple eate, Fy. (2) reduces to

$$
\varepsilon \frac{\partial c}{\partial t}+u \frac{\partial c}{\partial x}=0
$$

heving

$$
x=x_{0}+\frac{4}{\varepsilon} t
$$

as a chardeteristic curve. $c$ is constunt on a characteristic. There is no physical dispersion. kow, consider a finice differvence approximation to $\mathrm{Eq}$. (5).

$$
e \frac{c_{1}^{k+1}-c_{1}^{k}}{\Delta t}+u \frac{c_{i}^{k}-c_{i-1}^{k}}{\Delta x}=0
$$

The expression is chosen to employ upwind differeneing. The superscripts refer to the time level while the subscripts are spatial Indices, Using Taylor series expansions for the concentration, we nay readily show that $5 q$. (7) is equivalent to

$$
\varepsilon \frac{\partial c}{\partial t}+u \frac{\partial c}{\partial x}=\frac{u \Delta x}{2}\left(1-\frac{u \Delta t}{E \Delta x}\right) \frac{\partial^{2} c}{\partial x^{2}}+\cdots
$$

The coefficiest of the second spatial derivative on the right side is a numerical dispersion coefejcient, it results from the use of the finite difference expression. This confficient can exceed the actual dispersion coefficient by orders of magnitude.

Because the flow actually being considered is compressible, the situation is even more complox. The velocity varies with positiot and rime. Techniqurs teveloped lc elininate articial ditepersion in Eulerlat calculdtions, but relying on a uniform fluid veiocity, are not applicablo. Accordingly, a alxed EulerianLugrangian approach wa: considered and then adopted. In addition 10 the fixed Dulerion grid usted to calculate $\mu$, $u$, and 0 , a movine Laenanpiat mast. is uead to detemine elie conccitration, $L$.

h bagrangian ol:aerver moves, with J flaid element, along a clusucteritil it: curve of the cunvert ive trall:pror equation. Tine velocicy of a takrtingion moth point is

$$
\frac{d x}{d t}=\frac{u}{c}
$$

There is no conyect ive trantipor 
between Lagralugion mesh polnts. Such trajsport occirs solely as a result of dlspersion. The numerical dieporsion of the Eulerlan approach rosulted from the convective transport through the Eulorian weab. When a lagrangian viewpoint is adopted, there is no such transport and no numerical mechanism produeing such trangport is gencrated. The Lagrangian approzch provides a natural means of lininating artificial dispersion, valid for compressible flow and for flow with physical dispersion. fi Lagrangian technique has been usod by Garder, Peacenan, and Pozzi to treat dispersion in an incompressible flow through porous media. The technique described here differs by inc?uding conpressibility and also differs in the means of calculating dispersive transport.

\section{LAGRANGIAN FORNLATION}

A teluporal. derivative in the Larrangian frame is expressed by the waterial or substantial derivative.

$$
\frac{d}{d t}=\frac{\partial}{\partial t}+\frac{u}{E} \frac{\partial}{\partial x}
$$

so that the convective-dispersive transport equation, Eq. (2), becones

$$
c \frac{d c}{d t}+c \frac{\partial u}{\partial x}=\frac{\partial}{\partial x}\left(0 \frac{\partial c}{\partial x}\right)
$$

The first term on the left side of sq. (1I) Io the rate of change of concentration in a fluid element. This results fron dispersion, expressed on the right side, $t$. also frum compression or expansion of the fluid elenent as expressed in the second tern on the left.

As a result of this compressibility. the concentration is not constant on a wharactoristic curve even in the absence of dispersion. Additlonally, the spacing between successive losrangian mesh points will yary as they move throush the podius. Whither of these effects are present in in Incompressible flow,

\section{COWECTIV, RALSPORT}

First, let us addrons the problum of calculatine the positions of the points In tho nosun $1 f_{0}$ th expand iny Lasrongion meth. The Instunt uncous volocity of any Wegrangian meh polat, 4 , is glues by 7q. (9), The valocity, 4, ds given in terms of the pressure erodient by Li, (3). the problen roducer to one of interpolit.
Ing to obtaln the pressure grodfent at the locotion of $C$. The pressure is known at each of the Lulerilun nodes. These Eulerian nodes ara separated by a distance $\Delta x$. The Lagrangian node, $h$, is a distance, fax, in front of the nearest Eulerian node, i.

$$
|\mathbf{f}| \leq 1 / 2
$$

The pressures at the Eulerian nodes, $i-1,1$, and $1+1$, can be expresed in terns of a Taylor series expansion about k. These three expressions are then solved to yield the pressure gradicse, and thus the velocity, at the Lagrangian node.

$$
\begin{aligned}
\left.\frac{\partial p}{\partial x}\right|_{L}= & \frac{(2 f+1) p_{i+1}-4 f_{i}+(2 f-1) p_{i-1}}{2 \partial x} \\
& +\left.\frac{1-3 f^{2}}{6^{*}} \Delta x^{2} \frac{\partial^{3} p}{\partial x^{3}}\right|_{t}+\cdots \quad \text { (13) }
\end{aligned}
$$

Ilwerical integration of velocity ytelds the node position as a function of tine. Because the local velocity changes with position and time, however, the node velocity during a time interval $\Delta t$ is better approxinated hy a mean of calculated velocitieg at the two time levels and at the new and old positions. The ney position is unknow, however, until the calculation is complete. An iterative procedure to deternine the new position is employed. A single iteration appears to be adequate in our application. Such tine centering of lagrangian node notion calculations has previously been propossd by Forester. ${ }^{+}$

The concertration changer rosulting from expansiun of a fluid element may now be troated. This contribution to the variation of concentration is describes by Eq. (11) with dispersivity set eyuzl to zero.

$$
\epsilon \frac{d c}{d t}+c \frac{\partial u}{\partial x}=0
$$

The velocity eradient, ju/dx, i: positive in an expanding, flow and nept.sive in 3 comprosulns flow. it can be caltulatud by a varicty of methods. The methul recowanded hore, hedwer, is to nliminate the colculation untirely vith the followine oldiervition.

The bulk Fluld density olews a convective transport equalion an.llup?:aj!: to [q. (14), This la the continuity 
equation.

$$
c \frac{d p}{d t}+p \frac{\partial x}{\partial x}=0
$$

Consequently, the concentration of a fluid elevent changes in propertion with the density. Because the density in an isothermal ideal gas is proportional to the pressure, the concentration becores proportlonal to the pressure of the fluid elenent.

Because of dispersivity in the general case, the concentration does not gerain proportional to the pressure. Instead, the concentration change for a single time step is separated into convective ard dispersive ehanges. Starting with the concentration, $c k$ of of he fth Lagrangian node at tion level $k, t$. convective contribution yields an intermediate value of the new concentration.

$$
c_{l}^{k+1}=c_{q}^{k} \frac{P_{l}^{k+1}}{P_{l}^{k}}
$$

The intermediate spatial distrioution of concentration is then used to determine how dispersion ;" is the cencentration distribution at ti.s time level. Hote that, without explicitly introducing dispersion, no dispersion is generated.

The pressure used in Eq. (16) is found by a procedure similar to that used to obtain Eq. (13), the pressure gradient interpolation.

$$
\begin{aligned}
P_{l}= & \frac{f(t+1)}{2} P_{1+1}+\left(1-f^{2}\right) P_{I} \\
& +\frac{f(f-1)}{2} P_{i-1} \\
& -\left.\frac{1}{6} f\left(L-f^{2}\right) \Delta x^{3} \frac{d^{3} p}{d x^{3}}\right|_{l}+\cdots
\end{aligned}
$$

Havine accounted for node movement, expenslon, and compression, it remains to calculate the disporsive contriburion to transport of the tapged spocies.

\section{OISPCRSTVE TRAMSPORT'}

Diaponslve transpors is gounsmed by Eq. (II) with the fluid veloclty set equal to zoro.

$$
\varepsilon \frac{d \varepsilon}{d t}=\frac{\partial}{\partial x}\left(n \frac{\partial c}{\partial x}\right)
$$

mis is the diffusion equation. Its solution here is complicated only by the fact that the Lagrangian mest is nonunifort.

In order to find the concentrations at time level $k+1$, the lagranglan erid is held in its $k+1$ configuration. The interadiate concentrations, calculated to account for expansion and described in the previous section, are treated as the concentrations at time level $k$ but in the new positions. The order of operations is as if the node movement and fiuld expansion ocours instantoneously, then the tagged species disperses through stetionany fluld for a period of rine $\Delta t$.

The finite difference approximation to EA. (19) is developed in an analogous form to techniques widely used in the solution with a uniform mesh. For a uniform mash, one would write

$\varepsilon \frac{c_{l}^{k+1}-c_{l}^{k}}{\Delta t}=\frac{\theta[\delta(D \delta c)]^{k+1}+(1-g)[\delta(D \delta c)]^{k}}{\Delta x^{2}}$

$\sigma$ is the central difference operator. $B$ is a factor weighting the caleulation of the second spatial derivative betwee: time levels $k$ and $k+1$.

8 is zero for an explicit calculation. If $\beta$ is $1 / 2$ and $D$ is constant, this is the Crank-Hicolson method. for $B \geq 1 / 2$, the caleulation is uncondit ionally stable. Hote that the coefficient matrix of the unknown coneentrations is trigiagonal, so that the sane effisient algorithm ast wat employad to calculare pressure could be ured tere.

Then treating the nonuni form aceth. It is desirable to matain a tridideond form because of the considerable saving; In computationas timn. There is a pendilty In accuracy howner. A threrepoint upproximation to the :eecond drerivest isn, for exanple, kar a lawer omer irsor clian thr: uniform mesh syuivalent. 


$$
\begin{aligned}
\left.\frac{2^{2} \mathrm{~d}}{2 x^{2}}\right|_{l} & =\frac{2 c_{l+1}}{\left(x_{l+1}-x_{l}\right)\left(x_{l+1}-x_{l-1}\right.} \\
& -\frac{2 c_{l}}{\left(x_{l+1}-x_{l}\right)\left(x_{l}-x_{l-1}\right)} \\
& +\frac{2 c_{l-1}}{\left(x_{L}-x_{l-1}\right)\left(x_{l+1}-x_{l-1}\right)} \\
& -\frac{1}{3}\left(x_{l+1}-2 x_{l}+x_{l-1}\right) \\
& -\left.\frac{\partial^{3} c}{2 x^{3}}\right|_{l}+\cdots
\end{aligned}
$$

The leading error term vanishes for a unlform mesh. A nonunifor mesh should be generated so ss to maintain small values for these additional error terms. A smooth slow voriation of mesh size is best from this standpoint.

HIth varying dlffusivity, the nonuniform mesh equivalent of Eg. (19) was taken as

$$
\begin{aligned}
\varepsilon \frac{E_{l}^{k+1}-c_{l}^{k}}{\Delta t}= & \frac{2 B}{x_{l+1}-x_{l-1}}\left(p_{l+1} \frac{c_{l+1}-c_{l}}{x_{l+1}-x_{l}}\right. \\
& \left.=a_{l-k} \frac{c_{l}-c_{l-1}}{x_{l}-x_{l-1}}\right)^{k+1} \\
& +\frac{2\{L-t)}{x_{l+1}-x_{l-1}} \\
& =\left(b_{l+k} \frac{c_{l+1}-c_{l}}{x_{l+1}-x_{l}}\right. \\
& \left.-b_{l-k} \frac{c_{l}-c_{l-1}}{x_{l}-x_{l-1}}\right)^{k}
\end{aligned}
$$

apprash have been presented. Tochniques for accurately determining mes movement flutd expansion, and troce elenent dispersion are described. These techniques have been developed for the amalys is of transport associated with gas flows in porous media. A program, OLASPORA, employing these mathods has been written and implenented on the CDC $7600 \mathrm{~s}$ at the Larrence Liverwore Laboratony.

\section{REPERENCES}

1A. E. Scheideger, the Physics of Flon through Ponous Madia (Hachillan, New York, 1960)

IF. A. Horrison, Jr., I, E E. C. Fund. 11. 191 (1972)

'G. H. Bruce, D. K. Peaceman, H. H. Rachford, Jr., and J, O. Rice, Per. Trans. AIHE 198, 79 \{1953\}

3. Crank and P. Hicolson, Proc. Canb. Phil. Soc. 43, 50 (1947)

SD. U. von Rosenberg, Mettods for the Nunerical Solution of Partial Differential Squations (Anerican Elsevier, Ner York, 1969)

'A. 0. Carder, Jr., 0. H. Peacenan, and A. L. Pozzi, Jt., Pet. Tnans. AIHE 231, 26 (1964)

'c. K. Forester, J. Computational Phys. 12,269 (1973)

for 18 oqual to $1 / 7$, a sllght ouchtlation was oliserved to the results. A whoght lag facior, B, greater than J/7 j: recominended. No oscillution appesres for larger $\beta$.

\section{CONCLNSLONS}

Tho numerical creatinnt of convertivo ond divperijue trunsport has bein comildered. Rasone for utlilzing an Ealcrtuth-bagrimelan 
APPENDIX B

CONVECTIVE AND DISPERSIVE TRANSPORT IN A POROUS MEDIUN

R. S. Downing

F, A. Morrison, Ir. 


\title{
CONVECTIVE AND DISPERSIVE TRANSPORT IN A PORCUS MEDIUMA
}

\author{
R. S. Downing \\ F. A. Morrison, Jr. \\ Department of Hechanical and Industrial Ingineering \\ Un: rersity of Iilinois at Unbana-Champaign \\ Urbana, Il 61801
}

\section{ABSTRACT}

Convective and dispersive transport of a tagged species in a porous medium is investigated analytically. Tut flow is transient and compressible. As a means of avciding anomolous numerical dispersion in transpont calculations, an Eulerian-Lagcangian technique is developed. The Lagrangian mesh travels along characteristic curves at tie convective transport equation. Transport relacive to this mesh is dispersive. Detailed calculations are made for low Peclet number transport in the unsteady, low Reynolds number, isothemal flow of an ideal gas through the bed. Results are obtained for a bed of finite lentch. Similarity, valid in an infinite bed length, yields widely aprlicable results. The analysis and results are useful in the containment of underground nuclear explosions.

"This work was performed under the auspices of the USERDA and was supported by the University of Califomia Lawrence Livermore Laboratory under Subcontract 1160305 of Contract $\mathrm{H}-7405-$ Eng-48. 
INTRODUCTION

Following an underground nuclear explosion, gas from the cavity may enter the stamming column or surrounding porous mediun. Driven by the high pressure within the cavity, the fluid flows through the porous matenial. The duration period of this high cavity pressure is typically several minutes. The extent of penetration of cavity gas into the porous material is of interest in containgent evaluation.

Single phase [1] and multiphase [2] flow analyses have been presented describing such transient flows, If the absence of gaseous phase dispersion is posited, the gas originally in the medium is displaced in a piston-like manner. A distinct interface then exists between the gas originally in the bed and gas originating in the cavity. The extent of cavity gas penetration is unambiguous and can be cetermined in a straight forward manner. In any real flow, however, there is a dispersive transport as well as convective transport. A sharp interface does not exist. Instead, a gradual transition occurs. The purpose, then, of this paper is to analyze and describe the concentration distributions resulting from such transient flows with dispersion. Because the leading cavity Fluid is a gas, oniy an Ldeal gas flow is considered in detail. We do not examine the mutiphase flow following the leading cavity gas. 
THE TRAMSPORT EQUATION

We consider the transport of an inext trace species, characterized by a concentration $c$. The species is transported by convection and dispersion. Convective transport results from the directed motion of the canrier fluid. Without dispersion, there is no mixing. Changes in the species concentration within any fluid element would then result solely from compression or expansion of that element. The convective flux across a unit area normal to the direction of flow is

$$
j_{C}=\text { uc }
$$

$\mathrm{G}$ is termed the apparent velocity and is the volume flow rate per unit area.

The dispersion associated with miscible flow within porous media has received a great deal of attention. It is normally assumed that the dispersive flux can be described by Fick's law anö can be simply added to the convective flux. For the one-dimensional flow considered here, the dispersive flux is

$$
j_{D}=-D \frac{\partial C}{\partial x}
$$

$D$ is the dispersion coefficient and $x$ is a position coondinate in the direction of flow. In a two-dimensional flow, the dispersion coefficient for dispersion transverse to the direction of bulk fluid motion is, in general, different from the longitudinal dispersion coefficient. Only longitudinal dispersion is considered here.

The magnitude of the dispersion coefficient depends on the Peclet number, based on grain size, of the fluid flow. For low Peclet number flows, dispersion results primarily from molecular diffusivity. In this range, the dispersion coefficient is essentially independent of Peclet number and is of the onder of the molecular diffusion coefficient. In faster flows, additional mixing occurs as a result of the inhomogeneity of the medium. Velocity 
variations, path tortuosity, and dead spaces all contribute to increased dispersion. The dispersien coefficient is a monotonically increasing function of the Peclet nurber, being roughly proportional to Peclet number over a broad range. While theoretical madels for the dispersion coefficient are available, dimensionless correlations of experifiental results appear to have greater present utility. Reviews [3-4] of analysis and experiment directed toward providing hydrodynamic dispersion coefficients for porous media describe much of the work in this area.

The flows of particular interest here are characterized by Reynolds numbers less than one. Since gas Schnidt numbers are normally of order unity, the corresponding Peclet numbers are small. The dispersion coefficients are taken to be independent of the local velocity and to be of the order of magnitude of the molecular diffusion coefficient.

The molecular diffusion coefficient is itself a function of the thermodynamic state of the gas. The molecular diffusivity in gases increases with increasing temperature and varies inversely with the pressure [5]. Despite the high temperature within a nuclear cavity, the gas flow is isothermal at ambient temperature ahead of the saturation front produced by condensation [2]. While the gas and the solid material will rapidy come to local thermodynamic equilibrium, the heat capacity of the solid greatly exceeds that of the invading gas. Accordingly, the temperature rise from ambient in this portion of the porous medium will be small. Since the pressure will vary by at least an order of magnitude in the same region, the molecular diffusion coefficient, and thus the dispersion coefficient, is taken to vary only with pressure

$$
D=D_{0} \frac{P_{0}}{P}
$$


$p$ is the pressure, The subscript a denotes atmospheric conditions.

Conservation of species for an infinitesimal region of space jecomes

$$
\varepsilon \frac{\partial c}{\partial t}+\frac{\partial}{\partial x}\left(j_{C}+j_{D}\right)=0
$$

for a flow in one dimension. $\varepsilon$ is the porosity of the medium. $t$ is the time. Using the flux expressions, we have

$$
\varepsilon \frac{\partial c}{\partial t}+\frac{\partial}{\partial x}(u c)=\frac{\partial}{\partial x}\left(\partial \frac{\partial c}{\partial x}\right)
$$

the convective-dispersive transport equation in an Eulerian Erame. 
THE FLUID MOTION

In order to deternine the concentration distribution, the fluid velocity and pressure distributions must first be determined. The forms in which these distributions becone knom will also affect the subsegquent choice of tools utilizing them.

The fluid velocity in a low Reynolos number single phase flow is related to fluid and material properties by Darcy's law. The apparent velocity is

$$
y=-\frac{k}{\mu} \frac{\partial p}{\partial x}
$$

$k$ is the pemeability of the medium. $\mu$ is the fluid viscosity. Continuity for a compressible fluid is

$$
\varepsilon \frac{\partial p}{\partial t}+\frac{\partial}{\partial x}(\rho u)=0
$$

$\rho$ is the fluid density.

In an isothermel ideal gas, the visçsity may be taken as constant and the local density is directly proportional to the pressure. Combining (6) and (7), we obtain

$$
\frac{\partial}{\partial x}\left(p \frac{\partial p}{\partial x}\right)=\frac{g}{k} \frac{\partial p}{\partial t}
$$

governing the pressure. Permeability is presured uniform.

Prior to a nuciear explosion, the gas in the mediun is at a uniform ambient pressure, $F_{0}$. As a boundary condition, we will consider a step change to a higher pressure, $p_{1}$, at the inlet. The position, $x_{1}$ is taken as the distance from this inlet. Both semi-infinite and finite media are of interest. The results of analysis of flow in a semi-infinite medium may be applied to flow in a finite porous medium until such time as effects of the flow appear at the distant boundary. The colum length is denoted by 1 . 
For numerical calculation, it is convenient to introduce a set of dimensionless variabies and to express the goveming relations and results in terms of them. Dimenstonless position is

$$
x \equiv \frac{x}{\imath}
$$

ranging, in the finle bed, from sero at the inlet to one at the distant boundary. Dlaonsionless time is defined

$$
r \equiv \frac{k\left(p_{1}-p_{0}\right) t}{c \mu L^{2}}
$$

The rotic of applifed to infthal pressure is

$$
H=\frac{P_{1}}{P_{0}}
$$

in cinensionless pressure,

$$
P=\frac{p-p_{0}}{p_{1}-p_{0}}
$$

Jiso varying betwoen 0 and 1 , depends only on $x, T$, and $t$. The governing equat lon, (a), becones

$$
\frac{1}{2} \frac{\partial^{2}}{\partial u^{2}}\left(p^{2}+\frac{2 p}{y-1}\right)=\frac{\partial p}{\partial p}
$$

describing flow in a finite bed.

In an unbounded bed, the bed length is infinite and the separate definitions (g) and (10) are not sultable. The length is converiently renoved by the observation that the pressure distribution resulting from a seep pressure change at the surface of a semi-infinite porvus medium may be expressed in terms of a single variable, $\theta$, rather than position and time separately. Introducing

$$
\theta \equiv \frac{x}{2 \sqrt{7}}=\frac{x}{2} \sqrt{\frac{c \mu}{x\left(p_{1}-p_{0}\right) t}}
$$


we find that the governing partial differential equation (13) becomes the ondinary differential equation

$$
\frac{d^{2}}{d \theta^{2}}\left(p^{2}+\frac{2 p}{N-1}\right)+4 \theta \frac{d p}{d \theta}=0
$$

Subject to the boundary conditions

$$
\begin{array}{lll}
P=1 & \text { at } & \theta=0 \\
P+0 & \text { as } & \theta+\infty
\end{array}
$$

The flow is similar. Wote that the characteristic length $L$ does not appear in $\theta$.

Elows govemed by (13), on altematively, (15) were analyzed in [1] and results presented. In the results to be presented here, all fluid flow calculations are nunerical solutions of (13). For short times, until changes occur at the distant boundary, the flow in a finite column is identical to that in the infinite column. Pressures found as a function of position and time, can be expressed as a function of the similarity variable by using (14). The early time flow is similar. Attention was devoted to the analysis of finite colunns beczuse the results are nore general and because semi-infinite bed resilts are readily extracted from short time finite colum calculations. Furthermore, a reduction in the number of variables, producing an ordinary differential equation, does not necessarily simplify the aumerical solution. It may be noted, in this context, that boundary conditions (16) include a condition at infinite $\theta$.

Equation (13) is analogous to a nonlinear heat conduction equation. The numerical procedure selected for its solution is the method of Bruce, Peaceman, Rachford, and Rice [6]. The procedure is implicit and unconditionally stable. It is similar to the Crank-Nicolson [7] method for solution of the linear diffusion equation. Because (13) is nonlinear, the Bruce, Peaceman, Rachford, and Rice procedure is iterative. Its convergence is rapid. Horeover, the 
coefficient matrix of the finite difference equations is tridiagonal so that each iteration is efficiently performed. An algoritim [8] for the solution of a tridiagonal set of $n$ equations requires only $5 n-2$ storage locations and a aunber of operations of order $n$. 
TRAISPORT III LOW REYHOLDS NUABER HLOW

Before proceeding, the convection-dispersion equation may also be put into dimensionless form. Sultable poraneters were introduced in the discussion of "Iuid motion. A dimensionless spead,

$$
U \equiv \frac{\mu l y}{x\left(P_{1}-P_{0}\right)}
$$

would be unity in an incompressible Darcy flow. A reduced soncentration is expressed in terms of the concentration of entering gas, $c_{1}$.

$$
c \equiv \frac{c}{c_{1}}
$$

The dimensionless dispersivity is defined by

$$
D \equiv \frac{\mu D}{k\left(p_{1}-p_{0}\right)}
$$

and expresses the relative importance of dispersive and convective transport. It is roughly the inverse of a Peclet number based on bed length. In terms of these additional variables, equation (5) becomes

$$
\frac{\partial c}{\partial \tau}+\frac{\partial}{\partial X}(U C)=\frac{\partial}{\partial X}\left(D \frac{\partial C}{\partial X}\right)
$$

The dimensionless speed, $U$, is the speed of a fluid element in the dimensionless coordinates. In dimensional coordinates, this speed is the "pore velocity"

$$
\frac{u}{\varepsilon}=\frac{-k}{\mu \varepsilon} \frac{\partial p}{\partial x}
$$

Changing variables yields

$$
U=-\frac{\partial P}{\partial x}
$$

Introducing this expressien for $U$ in terms of the dinensionless pressure gradient into transport equation (20), we note that this partial differential equation may be written as an ordinary differential equation in the same 
ginilarity variable as describes the pressure.

$$
\frac{d}{d \theta}\left(C \frac{d P}{d \theta}\right)+2 \theta \frac{d C}{d \theta}+\frac{d}{d \theta}\left(D \frac{d C}{d \theta}\right)=0
$$

Since the initial concentration throughout the medium is zero and since the inler concentration is given a step change to a constant value, the initial and boundary conditions are compatible with a similarity solution.

$$
\begin{array}{lll}
c=1 & \text { at } & \theta=0 \\
c+0 & \text { as } & \theta+\infty
\end{array}
$$

The concentration distribution in the semi-infinite mediun is simblar and is expressed in terms of the same variable, $\theta$, as is the pressure. Altiough strictly valid only for semi-infinite medium, the sinilarity may be used With great accuracy in a finite bed until such time as effects of the flow appear at the distant boundary.

A finite colum requires an examinatiun of conditions to be inposed at: the distant boundary. For an open colum, the exit pressure is taken to be equal to the initial pressure. The solution of (14) reguires such a boundary condition as well as the initial condition and inlet boundary condition previousily imposed. In the absence of dispersion, no exit boundary condition is required for the solution of (20). With dispersion, however, a boundary condition becomes recessary. The form of this exit boundamy condition on concentration is not obvious. Convective and dispersive fluxes within the porous bed and directed towand this surface must be coupled to fluxes leading from this sumface and outside the bed. Test conditions beyond the medium are subject to considerable variation. Horeover, containnent efforts seek to predict, and then prevent, any measurable concentration of cavity gas at: the surface. Accordingly, we simply take as this boundary condition the widely used Danckwerts [9] condition for the exit of a chemical reactor. The dispensive flux, given by (2), is set equal to zero at the exit. 
This condition has been extensively employed in both steady state and unsteady state analyses. As noted by Hehner and Wilhelm [10] however, its use in the unsteady state is strictly valid only when the Peclet number is infinite in the region beyond the bed. 
MUERICAL DISPERSIOH

Locause an Eu?erian approach is used to detemine the velocity, pressure, and dispersivity distributions, Eulerian methods should be considered for subsequent analysis of trace component transport, Culerian techniques, however, tend to produce an anomolous numerical dispersion which can exceed the physical dispersion of interest here. Noh and Protter [11] analyzed the diffusion resulting from a finite difference approximation to the linear convection equation.

Consides briefly, as an example, the linear convective transport in an incomprassible flow. In this simple case, equation (20) reduces to

$$
\frac{\partial C}{\partial x}+u \frac{\partial C}{\partial x}=0
$$

with a constant velocity, $U$, and having

$$
x=x_{0}+U t
$$

as a characteristic curve. The concentrotion, $C$, is constant on a characteristic. There is no physical dispersion.

Further, consider a finite difference approximation to (25) which is forward in time and exploys upwind differencing.

$$
\frac{c_{i}^{k+1}-c_{i}^{k}}{\Delta t}+0 \frac{c_{i}^{k}-c_{i-1}^{k}}{\Delta x}=0
$$

The index denoting the time level is the superscript $k$. The subscripts, i; are spacial indices in the unform Eulerian mesh. Using Taylon series expansions for the concentration, neglecting terms above second order, and using (25), we find that (27) is equivalent to

$$
\frac{\partial c}{\partial \tau}+v \frac{\partial c}{\partial X}=\frac{v \Delta x}{2}\left(1-\frac{v \Delta \tau}{\Delta x}\right) \frac{\partial^{2} c}{\partial x^{2}}+\ldots
$$

The coefficient of the second spatial derivative on the right-hand side is a munerical dispersion coefficient. It has no physical basis but arises 
from the use of the finite difference expressions. Nunerical dispersion coefficients of this type can exceed the actual dispersion coefficient by orders of magnitude. For snall courant number, $14 t / \Delta x$, the ratio of these terms is roughly UAX/D which we siall call the "cell Peclet number." $D_{0}$ is the dimensionless dispersivity at ambient conditions.

Because the flow actually being investigated is transient and compressible and possesses physical dispersion, the situation is considerably nore complex. The velocity varies with position and time. Techniques developed to reduce artificial dispersion in Eulerian calculations, $b$ it relying on a uniform fluid velocity, e.g. [12], are not ayplicable. Accordingly, a mixed Eulerian-Lagnangian approach was considered, developed, and then adopter, In addition to the fixed uniform Euterian grid used to calculate pressure, velocity, and the dispersion coefficient, a moving nonuriform Lagrangian mesh is used to determine the concentration, $c$, of the transported trace component. The method yields accurate results independent of cell Peciet nunber. 


\section{LAGRANGTAN FORHULATION}

A Lagrangian observer moves, with a fluid element, along a characteristic curve of the convective transport equation. The velocity of a Lagrangian mesh point is, in dinensionless coordinates,

$$
\frac{d x}{d t}=v
$$

There is no convective transport between Lagrangian cells. Such transport occurs solely as a result of dispersion. The numerical dispersion of the Eulerian approach resulted from convective rransport through the Eulerian mesh. When a Lagrangian viewpoint is adopted, there is no such transport and no numerical mechanism producing such transport is generated. The Lagrangian approach provides a netural means of eliminating artificial dispersion, valid for compressible flow and for flow with phipical dispersion. A Legrangian technique has been used by Garder, Peaceman, and Pozzi [13] to traat dispersion in an incompressible flow through porous media. Their analysis did not address the myriad effects of compressibility. The technique described here diffens in that rospect, includes variable dispersivity, and differs in the means of calculating dispersive transport.

A temporal derivative in the lagrangian frame is expressed by the material on substantial derivative

$$
\frac{d}{d \tau}=\frac{\partial}{\partial \tau}+v \frac{\partial}{\partial X}
$$

So that the convective-dispersive transport equation, (20) becones

$$
\frac{d C}{d \tau}+c \frac{\partial U}{\partial X}=\frac{\partial}{\partial X}\left(D \frac{\partial C}{\partial X}\right)
$$

The first term on the left-hand side of (31) is the rate of change of concentration in a fluid element. This concentration change results from dispersive transpont into the fluid element, expressed on the right-hand side, 
and also, when the flow is compressible, from the compression or expansion of the fluid element. This last contribution is expressed in the second term on the left-hand side of (31).

As a consequerice of this compressibility, the concentration is not constant on a characteristic curve even in the absence of dispersion. Additionally, the spacing between successive Lagrangian mesh points will vary as they travel through the medium. Neither of these effects are present in an incompressible flow. 
CONVECTIVE TRANSPORT

First, let us address the problem of calculazing the positions of the points in the nonuniforn expanding Lagrangian mesh. The instantaneous velocity of any Lagrangian mesh point, $l$, is given by (29). In a low Reynolde number flow, this velocity is given in terms of the pressure gradient by equation (22). The problem then reduces to one of interpolating to obtain the pressure gradient at the location of $h$. The pressure is lonokn, from fluid flow calculations, at each of the Eulerian nodes. These Eulerian nodes are separated by intervals of $\Delta \mathrm{X}$ and the Lagrangian node, $l$, is 10cated at some distance, $f \Delta x$, in front of the nearest Eulerian node, $i$.

$$
|f| \leq \frac{1}{2}
$$

Using the pressuros at three Eulerian nodes, $i-1, i$, and $i+1$, the pressure gradient, and thus the velocity, at the bagrangian node is found with enron of onder $\Delta x^{2}$.

$$
\left.\frac{\partial P}{\partial X}\right|_{\ell}=\frac{(2 f+1) P_{i+l}-4 f P_{i}+(2 f-1) P_{i-1}}{2 \Delta x}-\left.\frac{1-3 f^{2}}{6} \Delta x^{2} \frac{\partial^{3} p}{\partial x^{3}}\right|_{\ell}+\ldots
$$

Nunerical integration of velocity yields the Lagrangian node position as a function of time. Because the local fluid velocity varies with position and time, however, the node velocity during a time interval $\Delta t$ is better approximated by a mean of calculated velocities at the two time levels and at the old and new positions. Since the new position is unknown until the calculation is complete, an iterative procedure to determine the new position is employed. A single iteration appears to be adequate in our application. A criterion used to judge the adequacy of the convective transport calculations is described in a later section on application and results. Time centering of Lagrangian node motion calculations has previousiy been proposed by Forester [14]. 
We may now consider the concentration changes resulting from the expansion or compression of a fluid element. This contribution to the variation of concentration is described by the convective transport equation, (31) with dispersivity set to zero.

$$
\frac{d C}{d \tau}+C \frac{\partial U}{\partial X}=0
$$

The velocity gradient, $\partial v / \partial x$, is positive in an expanding flow and negative in a compressing flow. It can be calculated by a variety of methods. The method recommended here, however, is to eliminate the calculation entirely with the following ooservation.

The bulk fluid density obeys a convective transport equation identical to (34). The continuity equation, (7), in a Lagrangian frame and written in dimensionless coordinates is

$$
\frac{d p}{d \tau}+\rho \frac{\partial U}{\partial X}=0
$$

The concentration of a fluid element changes in proportion to the density. Because the density in an isothermal ideal gas is proportional to the fluid pressure, the concentration becomes proportional to the pressure of the fluid element. In aimensionless form, this becomes

$$
C \sim(\mathbb{N}-1) P+1
$$

The pressure at Lagrangian node, $\ell$, is found by interpolating between the Eulerian nodes where the pressure is known.

$$
P_{l}=\frac{f(E+1)}{2} P_{i+1}+\left(1-f^{2}\right) P_{i}+\frac{f(E-1)}{2} P_{i-1}-\left.\frac{1}{6} f\left(l-f^{2}\right) \Delta x^{3} \frac{\partial^{3} P}{\partial x^{3}}\right|_{l}+\ldots
$$


The ernor is of order $\Delta x^{3}$. Equation (33) for the pressure gradient is simply a derivative of (37).

In the general case of convective-dispersive transpon, the concentration, because of dispersivity, does not remain proportional to the pressure. Instead, the concentration change for a single time step is separated into convective and dispersive changes. Starting with the concentration, $c_{l}^{k}$, of the $\ell$ th Lagrangian node at time level $\mathrm{k}$, the convective contribution yields an intermediate value of the new concentration at the succeeding time level.

$$
c_{l}^{k+1}=c_{l}^{k} \frac{(N-1) P_{l}^{k+1}+1}{(N-1) P_{\ell}^{k}+1}
$$

This internediate spatial distribution of concentration is then used to determine how dispersion alters the concentration distribution at this time level. Wc that, without explicitly introducing dispersion, no dispersion is generated. In the Lagrangian calculation of convective transport, a moving node with zero concentration at any time has zero concentration for all time. 


\section{DISPERSIVE TRANSPORT}

Dispersive transport is governed by the diffusion equation, equation $(20$ !, with the fluid velocity set equal to zero.

$$
\frac{d C}{d \tau}=\frac{\partial}{\partial X}\left(D \frac{\partial C}{\partial X}\right)
$$

Its solution here is complicated only by the fact that the Lagrangian mesh is nonuniform.

In onder to find the concentration at time level $k+1$, the Lagrangian grid is hold in its $k+2$ configuration. The intertiediate concentrations, calculated tu account for expansion and described in the previous section, are treated as the concentrations at time level $\mathrm{k}$ but in the new positions. The order of operations is as if the node movement and fluid expansion occur instantaneously, then the tagged species disperses through the stationary fluid during the time in erval $\Delta \tau$.

The finite difference approximation to the diffusion equation (18) is developed ir: an analogous form to techniques widely used in the solution for a uniform mesh. For a uniform mesh, one could write

$$
c_{l}^{k+1}=c_{l}^{k}=\frac{\Delta \tau}{\Delta x^{2}}\left\{\beta[\delta(D \delta c)]^{k+1}+(1-\beta)[\delta(D \delta C)]^{k}\right\}
$$

$\delta$ is the central difference operator. $B$ is a factor weighting the calculation of the second spatial derivative betreen levels $k$ and $k+1$.

$B$ is zero for an explicit calculation. For constant dispersivity, the behavion of $(40)$ is well known. Then $B$ is $1 / 2$, this is the Crank-Nicolson method having truncation error of order $\Delta \tau^{2}, \Delta x^{2}$. More generally, the error is of onder $\Delta \tau, \Delta x^{2}$. For $\beta \geq 1 / 2$, the calculation is unconditionally stable. Note that the coefficient matrix of the unknown concentrations is tridiagonal so that the same efficient algorithm as was used to calculate pressure could be used here. 
When treating the nonuniform mesh, it is desirable to retain a tridiagonal form because of the considerable savings in computational time. There is a penalty in accuracy, however. A three-point approximation to the second derivative, for example, has a lower onder error than the uniform mesh equivalent.

$$
\begin{aligned}
\left.\frac{\partial^{2} c}{\partial x^{2}}\right|_{l}= & \frac{2 c_{l+l}}{\left(x_{l+1}-x_{l}\right)\left(x_{l+1}-x_{l-1}\right)}-\frac{2 c_{l}}{\left(x_{l+1}-x_{l}\right)\left(x_{l}-x_{l-1}\right)} \\
& +\frac{2 c_{l-1}}{\left(\bar{x}_{l}-\bar{x}_{l-1}\right)\left(\bar{x}_{l+1}-x_{l-1}\right)}-\left.\frac{1}{3}\left(x_{l+1}-2 x_{l}+x_{l-1}\right) \frac{\partial^{3} c}{\partial x^{3}}\right|_{l}+\ldots
\end{aligned}
$$

The leading error term varishes for a uniform mesh. A nonuniform mesh should be generated so as to maintain small values for these additional error terms. A smooth slow variation of mesh size is rest from this standpoint.

With varying diffusivity, the nonuniform mesh equivalent of (40) was taken as

$$
\begin{aligned}
\frac{c_{l}^{k+1}-c_{l}^{k}}{\Delta \tau}= & \frac{2 \beta}{x_{l+1}-x_{l-1}}\left(D_{l+(1 / 2)} \frac{c_{l+1}-c_{l}}{x_{l+1}-x_{-}}-D_{l-(1 / 2)} \frac{c_{l}-c_{l-1}}{x_{l}-x_{l-1}}\right)^{k+1} \\
& +\frac{2(1-\beta)}{x_{l+1}-x_{l-1}}\left(D_{l+(1 / 2)} \frac{c_{l+1}-c_{l}}{x_{l+1}-x_{l}}-D_{l-(1 / 2)} \frac{c_{l}-c_{l-1}}{x_{l}-x_{l-1}}\right)^{k}
\end{aligned}
$$

$D_{\ell+(1 / 2)}$ is the dimensionless dispersivity midway between Lagrangian nodes $R$ and $l+1$.

For $\beta$ equal to $1 / 2$, a slight oscillation was observed in the results. Since the variable diffusivity and nonuniform mesh preclude the possibility of a higher order error associated with $B$ equal to $1 / 2$, there need be no 
reluctance to vary $\beta$. For stability, a weighting factor, $\beta$, greater than $1 / 2$ is recomnended. No oscillation has been observed for larger $\beta$. 
APPLICATION AND RESULTS

A program, DIASPORA, employing these muthods has been written and applied to the transient convective-dispersiva transport in a porous medium. Consider, as an example, the transpont resulting from a step increase in pressure and concentration at one end of a uniform porous bed. The bed is finite with the other end open to the atmosphere. The initial concentration in the bed is zero. The resulting pressure and concentration distributions are of interest in containment celculations. Pressure distributions have previo'sly been obtained for ideal gas [1] and multiphase [2] flows of this type, He here obtain the concentration distribution associated with the ideal gas flow.

Following [1] and [2], consider a flow characterized by a pressure ratio, $N$, of 45 . This value is obtained from 01sen's [15] description of the cavity pressure history of an underground nuclear explosion in alluvium, The pressure distribution in the bed, governed by (13), was found and is shown in Fig. 1. As the pressure increase propagates through the medium, the region of higher pressure is cleasiy discemible. A near discontinuity is present in the pressume gradient as a pressure front seems to travel through the bed. For higher pressure ratio, $\mathrm{N}$, the initial pressure rise at any location is even sharper, When the pressure ratio is infinite, this pressure rise is discontinuous. Because of this behavior, the far boundary has little effect on the flow for a vell defined period. For an infinite pressure ratio, the pressure front reaches the exit when $\tau$ is 0.38 . Prion to this time, the flow is similar and the pressure depends on the single variable, $\theta$, defined by (13), for large but finite pressure ratio, $\mathrm{N}$, the similarity is not rigorously exact but is an excellent approximation until $\tau$ is about 0.38 , This similarity can be seen 
in Fig. 1 where positions on the $\tau=0.2$ curve are twice the corresponding positions on the $t=0.05$ curve. The prossure calculation is Eulerian, using the procedure of Bruce, Peaceman, Rachford, and Rice [6].

The corresponcing concentration distribution in the absence of dispersion is presented in Fig. 2. The Lagrangian formutation yields the abrupt change in concentration as the injected gas passes through the columb. This feature would not be observed if an Eulerian expression such as (27) were employed. Note also that the early time behavion is very nearly similar. The effects of the distant boundary are not apparent for $\mathrm{T}$ less than about 0.39 . As With the pressure, 8 is the sole independent variable in a similar flox. The positions of corresponding points on the short time curves vany as the square soot of time. The agreenent anong the calculated concentration distributions in this regard is an excellent indication of the accuracy of the calculations.

The position of the interface is a particularly stringent and readily observed indication of this accuracy. When $\tau$ is 0.05 , the position of the interface was calculated to be 0.30 . Accordingly, the calculated value of $\theta$ at the interiace is, from (14), 0.67. Since the flow is nearly similar for time less than $0.38, \theta$ at the interface should remain relatively constant prior to this time. For times of $0.1,0.2$, and 0.3 , the calculated positions correspond to $\theta$ being $0.67,0.68$, and 0.68 , respectively. The initial step change in pressure at the interface is responsible for making this Lagrangian node the most difficult to move accurately.

The concentration curves for times 0.5 and 3.0 are shown ending before the exit. The reason is simply that the plotting routine used to generate the curves used the Lagrangian node nearest the exit as the final point. All leading nodes have exited in both cases. Because the concentr tion 
remains proportional to pressure in convective transport, the exit concentration $C$ is $N^{-1}$ or $45^{-1}$ for both curves.

Figures 3 through 5 show the effect of dispersivity. Variable dispersivity is employed in each case. At ambient pressure, the dimensionless dispersivity is $10^{-3}, 10^{-2}$, and $10^{-1}$, respectively, in these figures. The dimensionless dispersivity at ambient conditions $D_{0}$ can be estimated in any application using (19). Using air viscosity of $1.8 \times 10^{-5} \mathrm{~kg} / \mathrm{m} \mathrm{sec}$, a difrusivity of $2 \times 10^{-5} \mathrm{~m}^{2} / \mathrm{sec}$, a permeability of 0.1 darcy $\left(9.8 \times 10^{-14} \mathrm{~m}^{2}\right)$ and the applied pressure difference of $44 \times 10^{5} \mathrm{pa}$, the dimensionless dispersivity $D_{0}$ is of order $10^{-3}$. The dimensionless dispersivity would be larger in less permeable media, smaller in more permeable media. The permeability detemines the rate of convective transport. With convectivedispersive transport, application of the Danckwerts boundary condition, zero concentration gradient, at the exit yields the consentration there.

Each of the calculations presented used an Eulerian grid having 101 nodes. The time step was $2 \times 10^{-4 / 3}$ for 225 steps, tien changed to $10^{-3}$. The smaller initial time step is used to prevent large initial Lagrangian node movement. With either time step and presuming $U$ to bi of order unity, the numerical dispersion expressed by $(28)$ is of order $10^{-2}$. This numerical dispersion would be large compared to physical dispersion. The cell Peclet number is about 10 for the example just given. The simple Eulerian approach would not have yielded useful results. 


\section{CONCLUSIONS}

Convective and dispersive transport in transient compressible flow has been analyzed. The governing relations were formulated and a numerical procedure for calculating results was presented. Reasons for utilizing an Eulerian-Lagrangian approach were considered. Techniques for accurately ditermining mesh movement, fluid expansion, and trace element dispersion are described. Results were presented for transport resulting from a transient flow of an isothernal ideal gas through a uniform porous bed. 
IIGURE LEGENDS

Figure 1 Pressure distribution in unsteady flow

rigure 2 Concentration distribution in convective transport

Figure 3 Concentration distribution in convective-dispersive transport with varying dispersivity

Figure 4 Concentration distribution in convective-dispersive transport with varying dispersivity

Eigure 5 Concentration distribution in convective-dispersive transport with varying dispersivity 


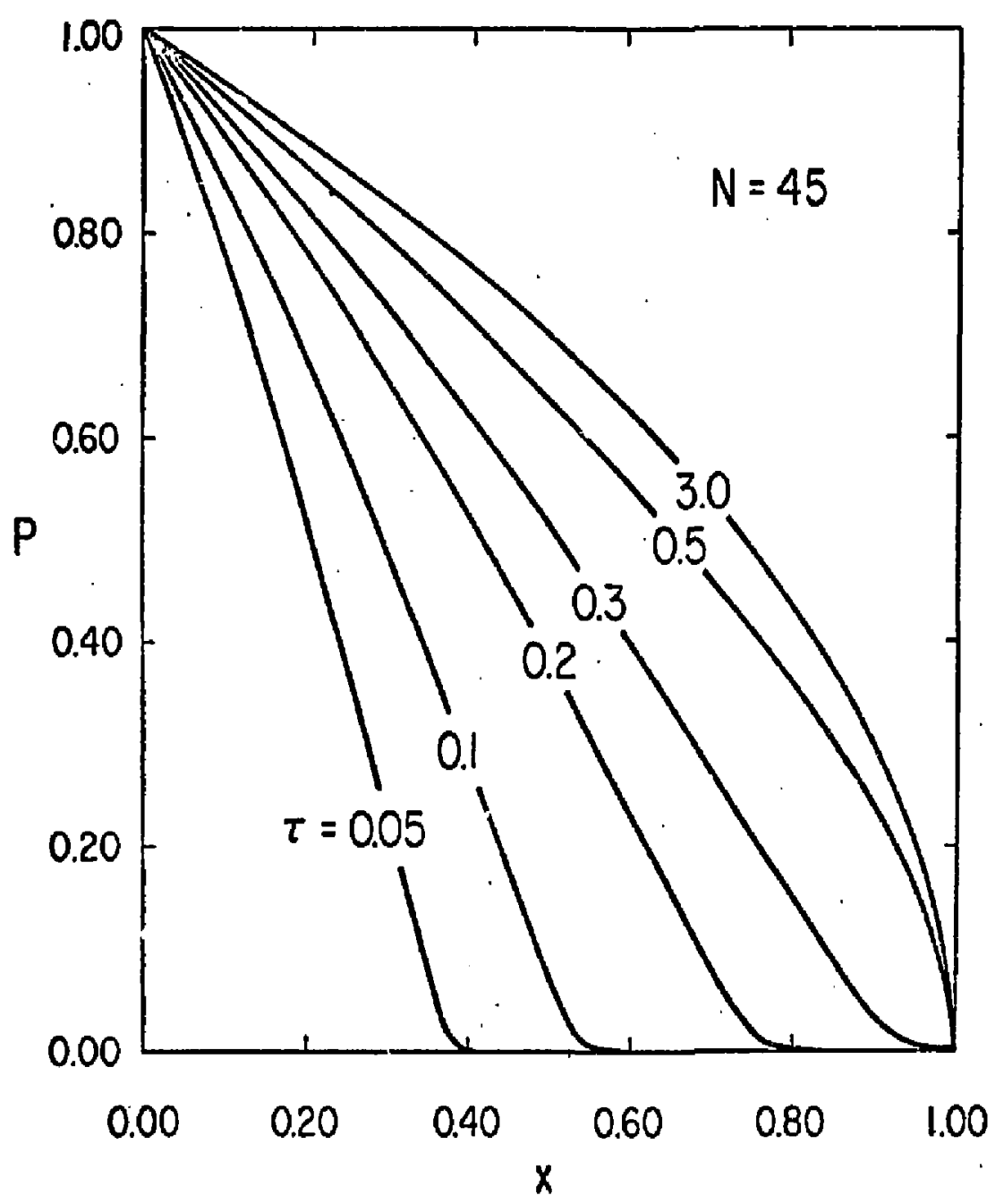

Figure 1 Pressure distribution in unsteady flow 


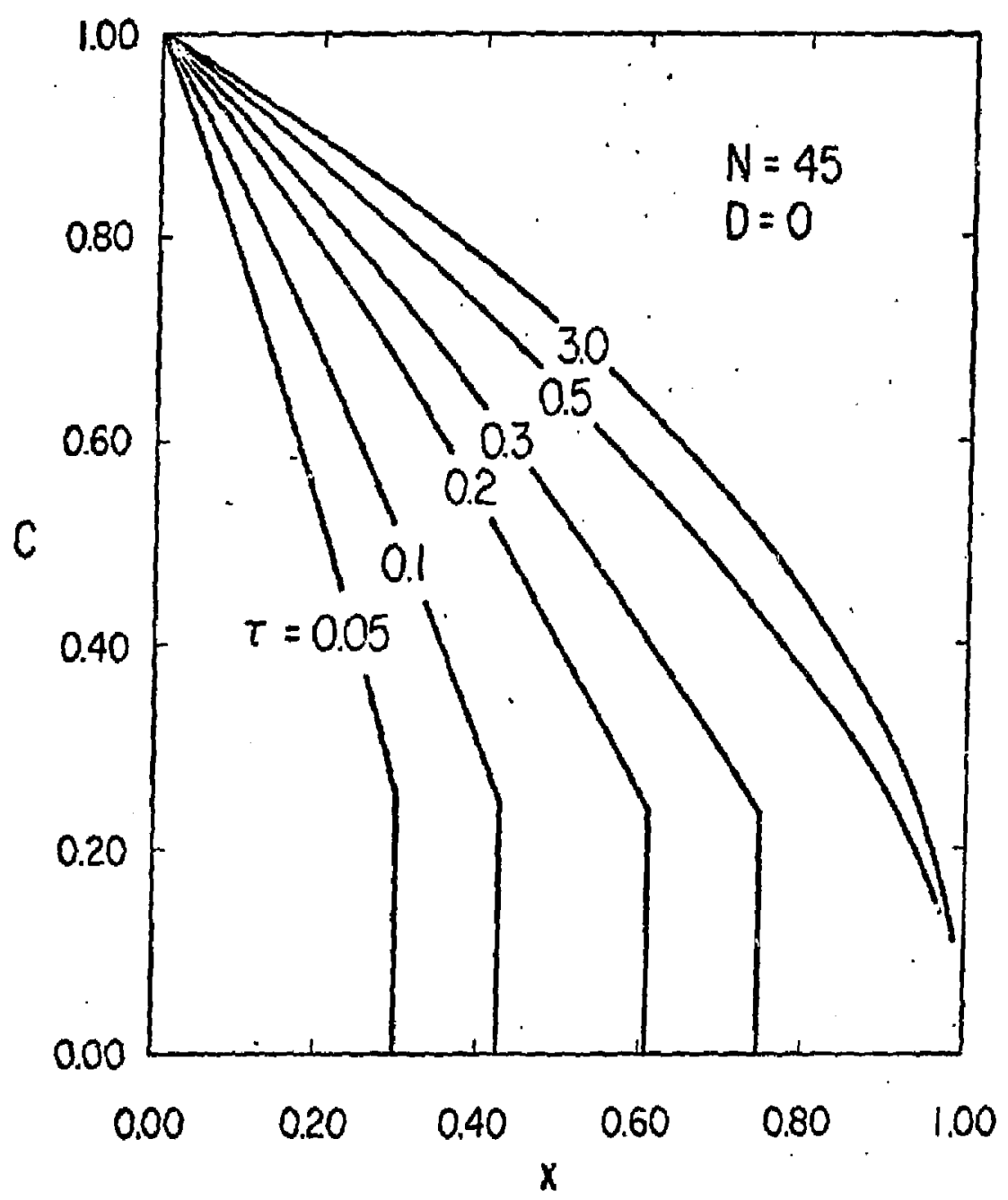

Figure 2 Concentration distribution in convective transport 


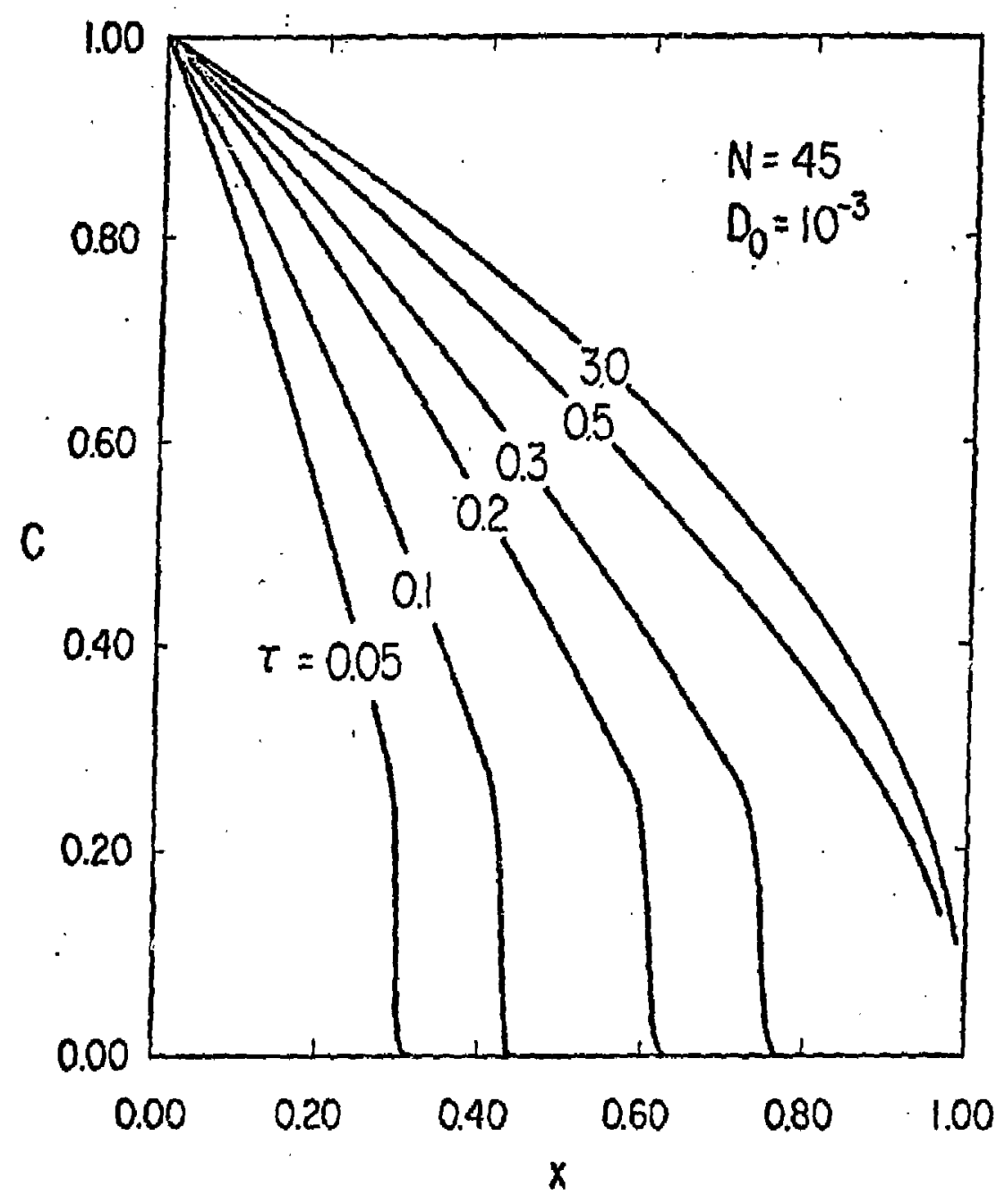

Figure 3 Concentration distribution in convectivedispersive transport with varying dispersivity 


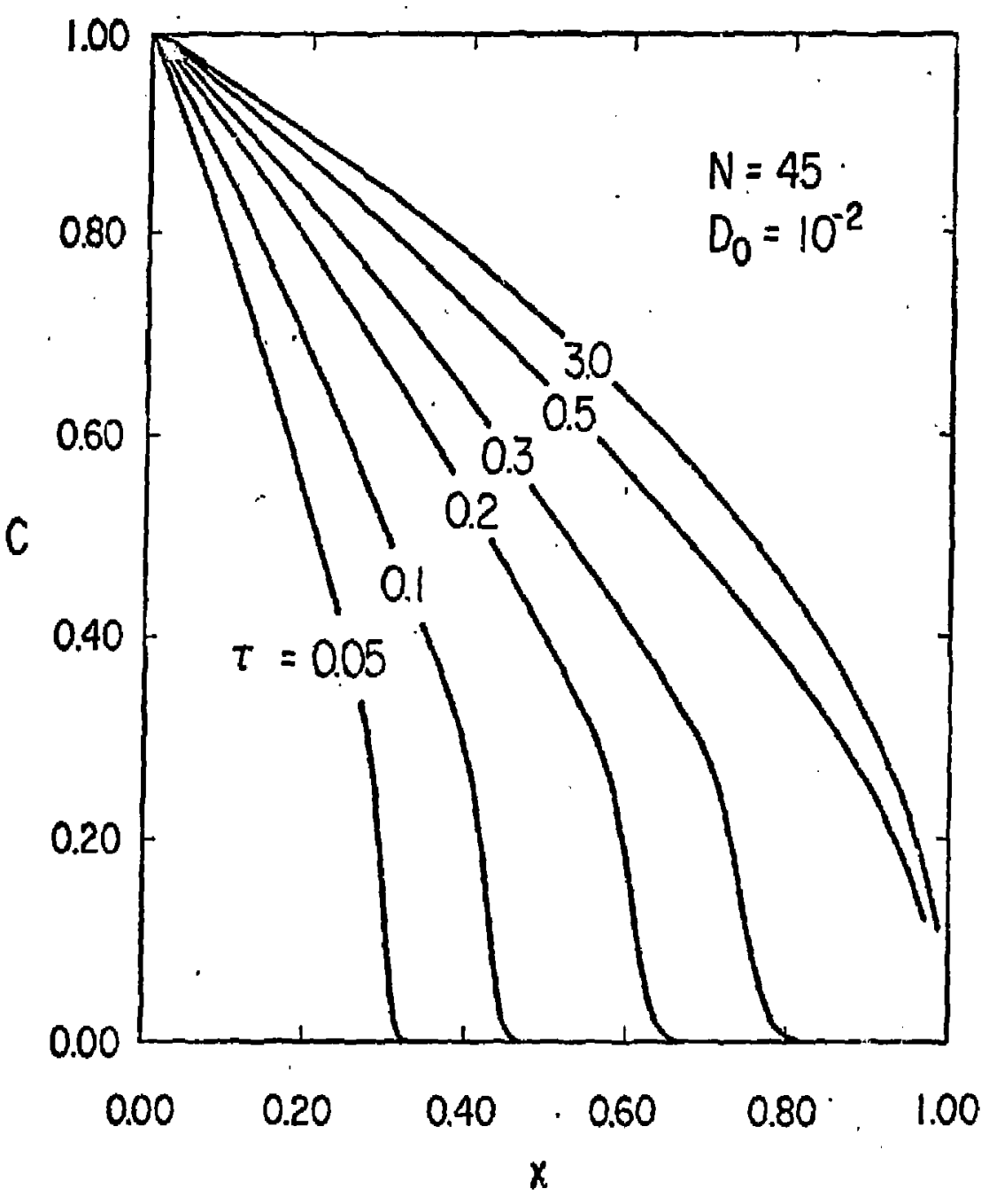

Figure 4 Concentration distribution in Convectivedispersive transport with varying dispersivity 


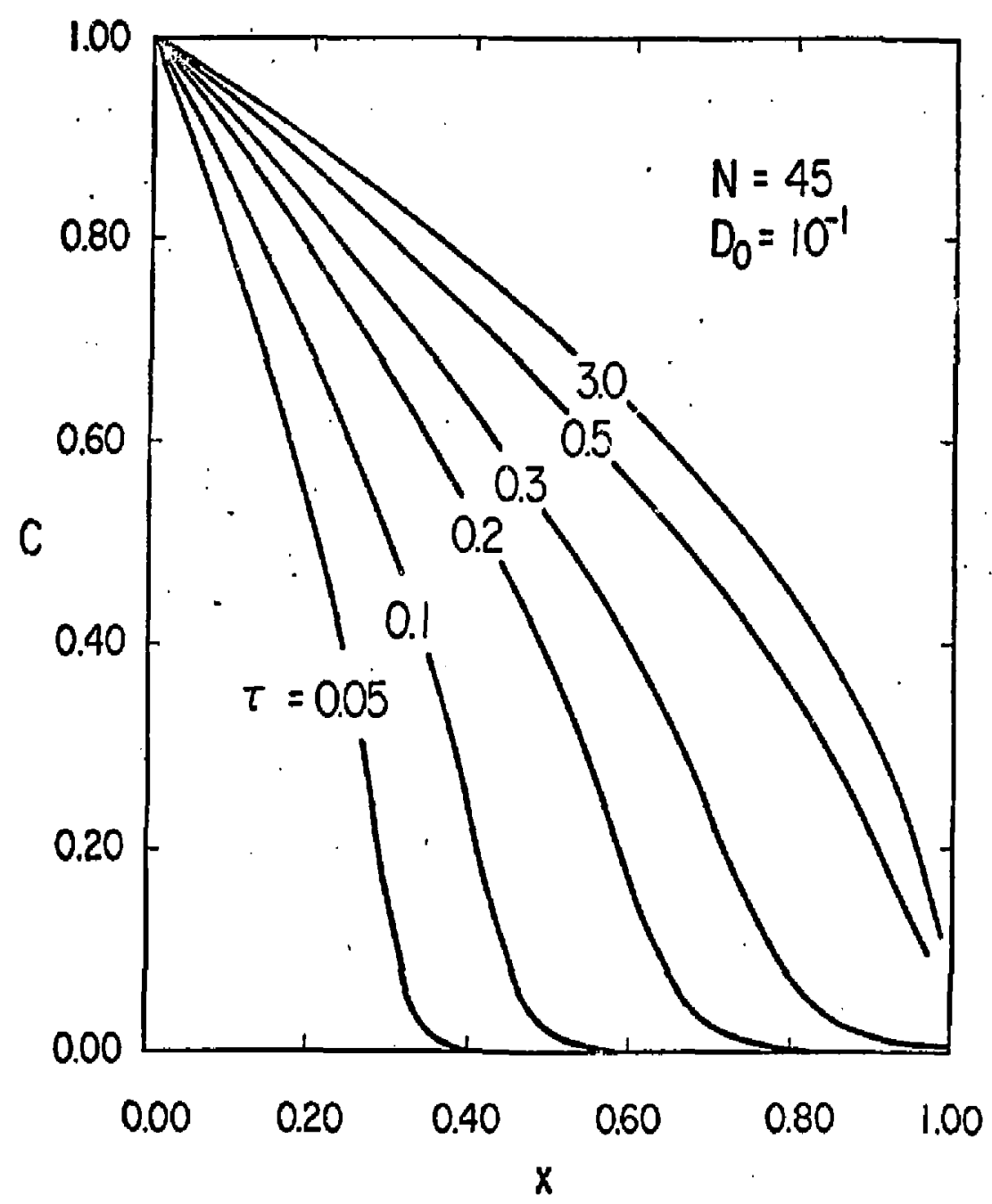

Figure 5 Concentration distribution in convectivedispersive trancport with varying dispersivity 


\section{REFERENCES}

2. E. A. Mormison, Jr., Transient gas flow in a porous colum, Ind. Eng. Chem. Fundam. 11, 191 (1972).

2. F. A. Morrison, Jr., Transient multiphase multicomponent flow in gorous media, Int. J. Heat Mass Inansfer, 16, 2331 (1973).

3. T. K. Perkins and O. C. Johnston, A review of diffusion and dispersion in porous media, Trans, Am. Inst. Min. Engrs, 22B, 70 (1963).

4. J. Dear, Dynamics of Eluids in Porous Media, American Elsevier, Nen York (1972).

5. J. O. Hirschfelder, C. F. Curtiss, and R. B. Bind, Molecular Theory of Gases and Liquids, Wiley, New York (1954).

6. G. H. Bruce, D. H. Peaceman, H. H. Rachford, Ir., and J. D. Ríce, Calculations of unsteady-state gas flow through porous media, Thons. Am. Inst. Min. Engrs. 198, 79 (1953).

7. J. Crank and P. Nicolson, A practical method for numerical evaluation of solutions of partial differential equations of the heat conduction type, Proc. Camb. Phil. Soc. 43, 50 (1947).

B. R. H. Hombeck, Numerical marching techniques for fluid flows with heat transfer, IIASA SP-297 (1973).

9. P. V. Danckwerts, Continuous flow systems, Chen. Eng. Sci, 2,1 (1953).

10. J. F. Wehner and R. K. Wilhelm, Boundary conditions of flow reactor, Chear. Eng. Sci. 6, 89 (1956).

11. W. F. Noh and M. H. Protier, Difference methods and the equations of tydrodynamics, J. Math. and Mech. 12, 149 (1963).

12. H, L, Stone and P, L, T, Brian, Numerical solution of convective transport problems, Am. Inst. Chem. Eng, J. 9, 681 (1963). 
13. A. 0. Garder, Jr., D. H. Feaceman, and A. L. Pozzi, Jr., Numerical calculation of multidimensional miscible displacement by the method of characteristics, Trans. Am. Inst. Min. Engrs. 231, 26 (1964).

14. C. K. Forester, A method of time-centering the Lagrangian marken particle computation, J. Comp. Phys, 12, 269 (1973).

15. C. H. Olsen, Time histchy of the cavity pressure and temperature following a nuclear detonation in alluvium, J. Geophys, Res, 72, 5037 $\{3967\rangle$. 
APPENUIX C

TRANSIENT NON-DARCY GAS FLOW IN A POROUS MEDLUM

f. ค. Horrison, Jr. 
TRANSIENT NON-DARCY GAS FLOW IN A POROUS MEDILW*

\author{
F. A. Morrison, Jr. \\ Meaber, SPE \\ University of Illinois at Urbana-Champaign \\ Urbana, IL 61801
}

\begin{abstract}
The high Reynolds number flow of gas through pocous materials is a subject of increasing significance. Gas recovery from fractured beds, gas flow produced by in oitu coal gasification, flows associated with storage and withdrawal in highly permeable structures, and transpiration cooling are examples. Darcy's law, normally used to describe flows through porous materials, is invalid in the range of Reynolds number of interest here. A nonlinear constitutive equation with empirically determined transpart properties is applicable instead.

Transient, compressible gas flow over a broad range of Reynolds number has been analyzed. The flow is governed by a set of coupled nonlinear partial differential equations. An iterative implicit stable numerical procedure has been developed and succussfully tested for calculations in one dimension. Results are given for the flow resulting from a step change in pressure at one end of a finite bed. A similarity solution is obtained for the flow into a seni-infinite bed. This latter solution is also applicable to short time flow in finite beds.
\end{abstract}

\footnotetext{
"This work was perforthed under the auspices of the USERDA and was supported by the University of California Lawrence Livermore Laboratory under Subcontract 1160305 of Contract $14-7405-$ Eng-48.
} 
INTRODUCTION

The high speed flow of a gas through a porous structure is a matter of considerable interest in sevirral areas. This interest has resulted -in a wealth of experimental infornation on such flows. In particular, Darcy's law, a linear constitutive equation between the apparent fluid velocity and the local pressure gradient, is found to fail for Reynolds numbers in excess of about 0.1 . Correlations, describing the deviations from Darcy's law, have been developed and have remarkable agreement among thenselves and with experiment.

The analytical ability to use these results in the prediction of more complex flows has not been obtained, however, While transient gas flows in the Darcy regime have been successfully analyzed and efficient algorithms for the calculation of multidimensional flows developed, little progress has been made in the nonlinear flow regime.

The purposes of this paper are to obtain relations governing nonDarcy, transient compressible flow, to develop a numerical procedure for calculations in one dimension, and to present results of the analysis of such a flow. 
DESCRIPTION OF THE FLOW

Consider the one-dimensional flow o: a gas through a porous structure. The apparent velocity of a fluid flowing through a porous bed is, by definition, the volume flow rate per unit area normal to the direction of flow. In a low Reynolds number flow, this velocity, $u$, is given by a linear constitutive equation, Darcy's law.

$$
u=-\frac{k}{\mu} \frac{\partial p}{\partial x}
$$

$k$ is the permeability of the medium, $\mu$ is the fluid viscosity, $p$ is the fluid pressure, and $x$ is the position coordinate in the direction of flow. For local Reynolds number, pud/ $\mu$, based on pore or grain size d and above about 0.1 , this relation is unsatisfactory. $\rho$ is the fluid density. Accause of the highly curved tortuous paths followed by fluid elenents, inertial effects become significant. The relation between velocity and pres. sure gradient becomes nonlinear.

Forchheimer [1]" proposed that Darcy's law be modified by the inclusion of a second order term in velocity. In the usual format, Forchheimer's relation is written.

$$
a_{1} u+a_{2} \rho u^{2}=-\frac{\partial p}{\partial x}
$$

Since this relation was originally proposed, an impressive anount of experimental evidence has been amassed to justify its use. In the low velocity range, the relation reduces to Darcy's law. The constant, $a_{1}$, is simply $\mu / k$ and can be determined experimentally or, for uniform beds, the widely used Carman-Kozeny relationship [2] or Happel's [3] free surface model yield Numbers in brackets refer to entries in REFERENCES. 
good estimates. At high velocities, the Forchheimer relation reduces to the form found experimentally by Burke and Plummer [4]. From the Darcy range, spanning a transition region, and up to Reynolds numbers of several thousand, the complete Forcheimer relation has accurately described results of experiments by numerous investigators. These include Ergun and Orning [5], Green and Duwez [6], Ergun [7], Schneebeli [8], and Ward [9]. Theoretical developments of the Forchheimer relation, e.g., Irmay [10], Bachmat [11], and Black [12], having varying degrees of sophistication are also avajlable.

A further generalization of (2) was proposed by PolubarinovaKochina [13] and consists of the inclusion of a local acceleration term.

$$
-a_{1} u+a_{2} p u^{2}+a_{3} p \frac{\partial u}{\partial t}=-\frac{\partial p}{\partial x}
$$

$\mathbf{t}$ is the time. Because disturbances propagate across a pore having a typical dimension d with a relaxation time of order $d^{2} / v$, this effect is negligible, $\downarrow$ is the kinematic viscosity. For air at atmospheric conditions, $y$ is $0.15 \mathrm{~cm}^{2} / \mathrm{sec}$. A bed with a permeability of one Darcy $\left(9.8 \times 10^{-9} \mathrm{~cm}^{2}\right)$ has a typical pore dimension of order $10^{-3} \mathrm{~cm}$. The corresponding relaxation time is of order $10^{-5} \mathrm{sec}$, considerably less than any significant time in a transient flow. Including this local acceleration effect has no obvious merit.

The effect of convective acceleration, as considered by Beavers and Sparrow [14], is expressed in the relation used by them. In a form consistent with our notation, they have

$$
a_{1} u+a_{2} p u^{2}+\frac{1}{\varepsilon^{2}} \rho u \frac{\partial u}{\partial x}=-\frac{\partial p}{\partial x}
$$

$E$ is the porosity of the medium, the vold volume fraction. The inertial coefficient, $a_{2}$, is of the order of the reciprocal pore dimension, $d^{-J}$. 
The length, $d$, is considerably smaller than any bed length of interest here. Variations in the apparent velocity occur over distances much larger than $d$. Accordingly, the quadratic resistance term greatly exceeds the convective acceleration contribution and the latter is safely neglected.

An excellent verification of this conclusion is provided by recent results of Masha, Beavers, and Sparrow [15], Compressible steady gas flow in one dimension was examined in a series of careful experiments where gas compressibility was significant. The porous medium was a block of foamed nickel with a mean pore size of roughty $0.05 \mathrm{in}$. and a length of $8 \mathrm{in}$. The length scale for velocity changes is this bed length. The relative importance of convective acceleration and inertial drag should then be approximately the ratio $0.05: 8$. The contribution of convective acceleration should, in this case, be roughly 0.6 percent of the effect of inertial drag. Masha, Beavers, and Sparrow found that experimental pressures and calculated pressures based on Eq. (4) agreed within 2 percent. They further found that calculations based on Eq. (2) normally agreed with those employing convective acceleration within 0.1 percent. At the highest test Reynolds number reported, 81.6, the deviation was about 0.7 percent. The two expressions agree within the expected range and both agree well with experiment. For larger beds, with correspondingly smaller pore size-to-bed length ratios, the difference between expressions will be smaller yet.

We conclude that Forchheimer's relation (2) adequately describes the resistance of transient compressible flow over a broad range of Reynolds number. Accordingly, this rolation is adopted and, in agreement with Darcy's law, written

$$
u+b \rho|u| u=-\frac{k}{\mu} \frac{\partial p}{\partial x}
$$


The quadratic term $u^{2}$ is here replaced by $|u| u$ to account for flow in either direction. $|u|$ is the absolute value of $u$. The coefficient $b$ can be determined from any of several correlations. Typicai are those of Ergun [7] and Hard [9] which yiels

$$
b=\frac{d}{85.7 \mu(1-\varepsilon)}
$$

and

$$
b=\frac{0.550(k)^{1 / 2}}{\mu}
$$

respectively.

In addition to the constitutive equation describing flow resistance, the flow obeys the continuity equation, an equation of state and the first law of therodynamics. Conservation of mass is

$$
\varepsilon \frac{\partial p}{\partial t}+\frac{\partial}{\partial x}(p u)=0
$$

for an incompressible porous structure. The ideal gas equation of state is

$$
\mathrm{p}=\mathrm{pRT}
$$

$R$ is the gas constant and $I$, the thermodynamic temperature.

Rather than exploying the first law of thermodynamics, it norraliy suffices to assume an isothermal flow. The relaxation time for theat transfer between the solid and gas is negligible compared with the :ime for the gas flow to respond to changes at the bed boundaries. Temperature equality between solid and gas is usually assumed. In a gas flow, the heat capacity of the gas in the void volume is considerably less than that of the surrounding solid. As a result, the solid temperature will nomally remain constant and the gas teaperature rapidly approaches this value. Hith the isothermal restriction, an ideal gas flow is governed by 
Eq. (5) and Eq. (8) which become

$$
u+(b / R T) p|u| u=-\frac{k}{\mu} \frac{\partial p}{\partial x}
$$

and

$$
\varepsilon \frac{\partial p}{\partial t}+\frac{\partial}{\partial x}(p u)=0
$$

Pressure and velocity are the sole independent variables.

Equivalent expressions for radial flow in cylindrical coordinates have been successfully employed $[16,17]$ to describe the performanie of natural gas wells. Transient high speed gas flow in one dimension has been examined gore recent!y [18]. In this latter case, however, the second-order differential equation describing the flow is incorrect as given. 
DIMENSIONLESS FORM

Because the governing equations, Eqs. (10) and (11), are nonlinear, their solution is most readily obtained using numerical techniques. Prior to developing a numerical procedure, it is advantageous to convert these exprissions to a dimensionless form. The dimensionless expressions are chosen to correspond closely with those used in the analysis of related low Reynolds number gas flow [19], low Reynolds number nultiphase flow [20] and low Reynolds number convective-dispersive transport [21].

The effects of inertial resistance can be observed in the response of an initially stationary gas to a sudden change of pressure at a boundary of a one-dimensional porous bed. A suitable dimensionless pres. sure, varying between zero and one, is

$$
P \equiv \frac{p-p_{0}}{p_{1}-p_{0}}
$$

$p_{0}$ is the ambient pressure while $p_{1}$ is the applied pressure.

A dimensionless position is expressed as a fraction of the bed length $L$.

$$
x \equiv \frac{x}{L}
$$

The distance is measured from the inlet boundary where the pressure $P_{1}$ is applied.

Dimensionless time, in terns of these and previously defined properties, is

$$
T=\frac{k\left(p_{1}-p_{0}\right) t}{\mu \varepsilon L^{2}}
$$

A low Reynolds number flow has a response time corresponding to $:$ of order unity. rermeability and porosity are presumed uniform. 
A dimensionless speed, defined by

$$
u \equiv \frac{\mu L u}{k\left(P_{l}-P_{0}\right)}
$$

expresses the ratio of the local velocity to the velocity that would be observed in an incompressible Darcy flow having the same applied pressure.

For convenience, we refer to the ratio of applied pressure to initial pressure by

$$
N \equiv \frac{p_{1}}{p_{0}}
$$

A Reynolds number characterizing the flow and expressing the importance of deviations from Darcy behavior is

$$
\operatorname{Re}=\frac{p_{0} k\left(p_{1}-p_{0}\right) b}{\mu L}
$$

This is roughly a Reynolds number based on initial density, Darcy velocity and a pore dimension.

In terms of these paraneters, Eqs. (10) and (11) become

$$
U+\operatorname{Re}[(N-1) P+1]|U| U=-\frac{\partial P}{\partial X}
$$

and

$$
\frac{\partial P}{\partial T}+\frac{\partial}{\partial X}\left[\left(P+\frac{1}{N-1}\right) u\right]=0
$$




\section{CHANGE OF SCALE}

The dimensionless variables as selected are suitable for description of low Reynolds number flow with large pressure ratio. Under other conditions, a change of time scale is advisable.

Consider first the Darcy flow described by Eqs, (18) and (19) with Re set equal to zero. Substitution yields tile single differential equation describing tine pressure

$$
\frac{\partial p}{\partial \tau}=\frac{1}{2} \frac{\partial^{2}}{\partial x^{2}}\left(p^{2}+\frac{2 p}{N-1}\right)
$$

When the pressure ratio is well in encess of one,gas in the Darcy flow regime will respond to changes in a time $t$ of order unity. Darcy flow in a finite bed will approach steady state in a time of this magnitude.

For very large Reynolds number, however, the inertial resistance dominates. Consider, for simplicity, flow in the positive direction so that the absolute value $|U|$ becomes $U$ and the pressure gradient is nogative. In this case and for large Re, Eq. (18) reduces to

$$
U^{2}=\frac{\frac{\partial P}{\partial X}}{\operatorname{Re}[(N-1) P+1]}
$$

and the pressure is governed by

$$
[\operatorname{Re}(N-1)]^{1 / 2} \frac{\partial P}{\partial \tau}+\frac{\partial}{\partial X}\left[-\frac{1}{2} \frac{\partial}{\partial X}\left(P^{2}+\frac{2 P}{N-1}\right)\right]^{1 / 2}=0
$$

Now, when $\mathrm{N}$ is large, the appropriate time scale is seen to be

$$
\tau \equiv \frac{\tau}{[\operatorname{Re}(N-1)]^{1 / 2}}=\left(\frac{k p_{0}}{\rho_{0} \varepsilon^{2} L^{3} b \mu}\right)^{1 / 2} t
$$

so that the pressure obeys

$$
\frac{\partial P}{\partial T}+\frac{\partial}{\partial X}\left[-\frac{1}{2} \frac{\partial}{\partial x}\left(P^{2}+\frac{\partial P}{S-1}\right)\right]^{1 / 2}=0
$$


For smali pressure ratio, examination of Eq. (20) Ieveals that the suitable time scale for Darcy flow is

$$
\frac{\tau}{N-1}=\frac{k p_{0} t}{\mu E L^{2}}
$$

while, for high Reynolds number and small pressure ratio, rearranging Eq. (24) indicates that a time scale

$$
\frac{T}{(N-1)^{1 / 2}}=\frac{\tau}{(N-1)(R e)^{1 / 2}}=\left[\frac{k p_{0}}{P_{0} \varepsilon^{2} L^{3} b \mu(N-L)}\right] t
$$

should be employed.

Choice of an appropriate time scale is important in numerical calculations. Without consideration of the relaxation time, finite difference time steps are not well chosen, Excessively large time steps result in loss of accuracy while calculations using overly smali time steps are inefficient. 


\section{SIMILARITY ANALYSES}

While our primary interest $s$ in flow in finite beds, it: is worthwhile to consider the flow into a semi-infinite bed. There are several reasons for this interest. The short time behavior of flow in a finite bed is well approximated by flow in a semi-infinite bed. Additionally, under certain conditions to be described, the transient pressure in a semi-infinite bed depends only on a single variable and not on position and time separately. Such a flow is said to be similar.

Similarity permits several general conclusions about early time behavior and also permits one to assess the accuracy of numerical solutions. This latter feature is particularly valuable when, as is the case here, the governing equations are nonlinear and exact solutions for comparison do not exist.

Consider a flow into a semi-infinite bed. The gas in the bed is initially stationary and at ambient pressure. The pressure at the surface is sudderly increased to some high constant value. The flow then is governed by Eqs. (18) and (19) subject to

$$
\begin{array}{lll}
P=0 & \text { at } & T=0 \\
P=1 & \text { at } & X=0 \\
P+0 & \text { as } & X+\infty
\end{array}
$$

For Darcy flow, governed by Eq. (20) and subject to these conditions, the prissure has been shown [19] to be sinilar. The pressure depends only on a single variable defined by

$$
\theta=\frac{x}{2(\tau)^{1 / 2}}=\frac{x}{2} \sqrt{\frac{\varepsilon \mu}{k\left(p_{1}-p_{0}\right) t}}
$$


sine's both the differential equation and boundary conditions can be written in terats of $\theta$ alone. Equation (20) is

$$
4 \theta \frac{d P}{d \theta}+\frac{d^{2}}{d \theta^{2}}\left(p^{2}+\frac{2 P}{N-1}\right)=0
$$

and conditions (2) become

$$
\begin{array}{lll}
P=1 & \text { at } & \theta=0 \\
P+0 & \text { as } & \theta \rightarrow \infty
\end{array}
$$

Note that $\theta$ does not contain a length scale $L$.

The pressure distribution for this Darcy gas flow in a semi-infinite bed was calculated by Morrison [19] and the results are shown in Fig. 1. Several values of pressure ratio have been selected. The Reynolds number is zero in each of these cases.

For high Reynolds number flow, we may also demonstrate similarity. The similarity variable is different however from the variable, $\theta$, that has beon used for the low Reynolds number flaw. Consider the flow goversed by Eq. (24j and subject to the conditions (27). If we define

$$
\xi=\frac{x}{T^{2 / 3}}
$$

then Eq. (24) can be written as the ordinary differential equation

$$
5 \frac{d P}{d \xi}-\frac{d}{d \xi}\left[\frac{-9}{8} \frac{d}{d \xi}\left(p^{2}+\frac{2 P}{N-1}\right)\right]^{1 / 2}=0
$$

and conditions (27) are expressed in tems of $\xi$ by

$$
\begin{aligned}
& P=1 \text { at } \xi=0 \\
& P+0 \text { as } \xi+\infty
\end{aligned}
$$

corresponding to the conditions (30) applied to Darcy flow. $\xi$, like $\theta$, contains no length $L$.

The high Reynolds number gas flow resulting from a step change in surface 
pressure bas been snown to be similar. The pressure depends only on $\xi$. The short time flow in a finite column will behave in this manner. This similarity requirement can be used to judge the accuracy of finite difference calculations. Such calculations are performed and displayed in similar form in the section on results of calculations. 
NUMERICAL TECINIQUL:

A numerical procedure has been developed for the solution of Eqs.

and (19) governing the transi ent nun-Darcy isothermal gas flow in one dimension. Because stability cursiderations may severely restrict the allowable time step in an explicit calculation, an implicit method was devel oped.

Explicit methods have been developed, e.g. [22-26], that are unconditionally stable for solution of the linear diffusion equation. It remains to evalunte the applicability of their analogs to the nonlinear set of equations treated here, Consistency requirements will, at least in certain cases, place limits on the allowable step sizes.

A uniform mesh is employed. The node spacing in the $X$ diroction is $\Delta X$ and the spatial index is $i$. The temporal step size is $\Delta \tau$ and the time level is denoted by the superscript $k$.

Suitable finite difference expressions for the derivatives may now be developed. For this purpose, it is useful to interpret $P_{i}$ as the dimensionless pressure at node $i$ and $u_{i}$ as the dimensionless velocity midway between rodes $i$ and $i+1$. The spatial derivative in Eq. (19) expresses the rate of mass flow into a spatial element. At node $i$, the derivative is approxinated by

$$
\begin{aligned}
\frac{\partial}{\partial X}\left[\left(P+\frac{1}{N-1}\right) U\right]= & \frac{1}{2 \Delta X}\left[\left(P_{i+1}+P_{i}+\frac{2}{N-1}\right) U_{i}\right. \\
& \left.-\left(P_{i}+P_{i-1}+\frac{2}{N-1}\right) U_{i-1}\right]
\end{aligned}
$$

The velocities in this expression may be expressed using a finite difference equivalent of Eq. (18).

$$
v_{i}=\frac{-1}{\Delta x} \frac{P_{i+1}-P_{i}}{1+\frac{(W-1)}{2}-\frac{R e}{\left.P_{i+1}+P_{i}+\frac{2}{N-1}\right)\left|u_{i}\right|}}
$$


The pressure gradient of Eq. (18\} has been replaced by a central difference approximation for the derivative midway between $i$ and $i+1$.

The finite difference approximation of Eq. (19) is formed using a forward difference in time and expressing the spatial derivative as a weighted average of finite difference expressions at the $k$ and $k+1$ levels. Using Eqqs. (34) and (35), we have

$$
\begin{aligned}
& \frac{P_{i}^{k+1}-P_{i}}{L \tau}=\frac{1}{2(\Delta X)^{2}}\left\{B \left[\frac{\left(P_{i+1}+P_{i}+\frac{2}{N-1}\right)\left(P_{i+1}-P_{i}\right)}{1+\frac{N-1 R e}{2}\left(P_{i+1}+P_{i}+\frac{2}{N-1}\right)\left|U_{i}\right|}\right.\right. \\
& \begin{array}{l}
\left.-\frac{\left(P_{i}+P_{i-1}+\frac{2}{N-1}\right)\left(P_{i}-P_{i-1}\right)}{1+\frac{(N-1) R e}{2}\left(P_{i}+P_{i-1}+\frac{2}{N-1}\right)\left|u_{i-1}\right|}\right]^{k+1} \\
+(1-\beta)\left[\left(P_{i+1}+P_{i}+\frac{2}{N-1}\right) u_{i}-\left(P_{i}+P_{i-1}+\frac{2}{N-1}\right) U_{i-1}\right]^{k}
\end{array}
\end{aligned}
$$

The spatial derivative is expressed differently at the two time levels for reasons that will shortly be apparent. $\beta$ is the weighting factor. It can assume values between 0 and $1 . \quad \beta$ equal to zero corresponds to an explicit formulation. $\beta$ greater than zero is implicit. By analogy with the linear diffusion equation, $B$ greater than or equal to $1 / 2$ can be expected to yield stable results, independent of time step. In the absence of rigorous stability linits for nonlinear equations, such anslogs provide useful guidance. The corresponding finite different approximation, with $\beta$ equal to $1 / 2$, is the Crank-Nicolson method [27]. The Crank-Nicolson method has a truncation error of $(\Delta \tau)^{2},(\Delta x)^{2}$, when $B$ 
is not equal to $1 / 2$, the truncation error is of order $\Delta \tau,(\Delta x)^{2}$. For the nonlinear equation treated here, $\beta$ equal to $1 / 2$ would not yicid higher order error so that the choice of $B$ is somewhat arbitrary. $B$ equal to $1 / 2$ has been satisfactory in applications to datc; hatever, he option of increasing $\theta$ is retained.

The nonlinear diffusion equation governing Darcy flow of a gas has been solved, using $\beta$ equal to $1 / 2$, by Bruce, Peaceman, Rachford, and Rice [28]. This procedure is efficient, iterative, and stable. It has been widely employed.

Defining

$$
h=\frac{(\Delta x)^{2}}{\Delta \tau}
$$

and rearranging with the unknowns, the variables at the $k+1$ time level, on the left and known quantities, those evaluated at the $k$ time level, on the right.

$$
\text { 4h } \begin{aligned}
P_{i}^{k+1}-2 B & {\left[\frac{\left(P_{i+1}+P_{i}+\frac{2}{N-1}\right)\left(P_{i+1}-P_{i}\right)}{1+\frac{N-1) R e}{2}\left(P_{i+1}+P_{i}+\frac{2}{N-1}\right)\left|u_{i}\right|}\right.} \\
& \left.-\frac{\left(P_{i}+P_{i-1}+\frac{2}{N-1}\right)\left(P_{i}-P_{i-1}\right)}{1+\frac{(N-1) \operatorname{Re}}{2}\left(P_{i}+P_{i-1}+\frac{2}{N-1}\right)\left|u_{i-1}\right|}\right]
\end{aligned}
$$

$$
\text { 4h } P_{i}^{k}+2(1-\beta)\left[\left(P_{i+1}+P_{i}+\frac{2}{N-1}\right) U_{i}-\left(P_{i}+P_{i-1}+\frac{2}{N-1}\right) U_{i-1}\right]
$$


This relation, to be solved for the pressures at the new time level, is nonlineas in the unkrowns. The pressures are obtained by linearization and iterative solution of the resulting set of linear equations. The nonlinear porting is factored and then linearized by assuming a value for part of the expression. Because the assumed value may not be presumed correct, an iterative procedure is employed to generate progressively better assumtions.

Using the superscript $k+1$ to denote assumed values at level $k+1$, Eq. (38) is linearized in a manner consistent with, but generalizing, the Linearization of Bruce, Peaceman, Rachford, and Rice.

$$
\text { 4h } \begin{aligned}
p_{i}^{k+1} & =2 \beta\left[\frac{P_{i+1}+P_{i}+\frac{2}{N-1}}{1+\frac{N-I) R e}{2}\left(P_{i+1}+P_{i}+\frac{2}{N-1}\right)\left|u_{i}\right|}\right]^{K+1}\left(P_{i+1}-P_{i}\right)^{k+1} \\
& +2 B\left[\frac{P_{i}+P_{i-1}+\frac{2}{N-1}}{1+\frac{N-1) R e}{2}\left(P_{i}+P_{i-1}+\frac{2}{N-1}\right)\left|u_{i-1}\right|}\right]^{K+1}\left(P_{i}-P_{i-1}\right)^{k+1} \\
& =4 h P_{i}^{k}+2(1-\beta)\left[\left(P_{i+1}+P_{i}+\frac{2}{N-1}\right) u_{i}-\left(P_{i}+P_{i-1}+\frac{2}{N-1}\right) u_{i-1}\right]^{k}
\end{aligned}
$$

We have a set of linear equations among the unknown pressures. In a more compact notation, this is

$$
A_{i} p_{j-1}^{k+1}+B_{i} p_{i}^{k+1}+C_{i} p_{i+1}^{k+1}=D_{i}
$$

where 


$$
\left.\begin{array}{l}
A_{i}=-2 \beta\left[\frac{P_{i+1}+P_{i}+\frac{2}{N-1}}{1+\frac{(N-1) R e}{2}\left(P_{i+1}+P_{i}+\frac{2}{N-1}\right)\left|U_{i}\right|}\right]^{k+1} \\
C_{i}=-2 \beta\left[\frac{P_{i}+P_{i-1}+\frac{2}{N-1}}{1+\frac{(N-1) R e}{2}\left(P_{i}+P_{i-1}+\frac{2}{N-1}\right)\left|U_{i-1}\right|}\right]^{k+1} \\
B_{i}=4 h-A_{i}-C_{i} \\
D_{i}=4 h P_{i}^{k}+2(1-\beta)\left[\left(P_{i+1}+P_{i}+\frac{2}{N-1}\right) U_{i}-\left(P_{i}+P_{i-1}+\frac{2}{N-1}\right) U_{i-1}\right.
\end{array}\right]^{k}
$$

The sct of finite difference equations ( $46 j$ has a tridiagonal coefficient matrix and thus the iterations can be done efficiently. The solution of a set of $n$ such equations can be obtained using a well known algorithm, described by Bruce, Peaceman, kachford, and Rice [28], requiring only $5 n$ - 2 storage lacations and using a number of operations of order $n$.

If $\beta$ is chosen equal to zero, the pressures are uncoupled, the formulation is simpler, the method is explicit but stability requirements restrict the temporal step size $\Delta \tau$ to the order of $(\Delta x)^{2}$. Within this restristion, the explicit formulation has also been successfully used by the author. Thr restriction vanjshes when $\beta$ greater than $1 / 2$ is used. 


\section{RESULTS OF CALCULATIONS}

A series of calculations have been perficmed using the implicit procedure just described. Transient response characteristics are examined by considering the flow produced by a stop change in pressure at one end of a finite bed. The other end remains open to the atmosphere, its pressure unchanged. The flow, thon, is governed by Eqs. (18) and (19) subject to

$$
\begin{array}{lll}
P=0 & \text { at } & T=0 \\
P=1 & \text { at } & X=0 \\
P=0 & \text { at } & X=1
\end{array}
$$

Only the pressure ratio $N$, defined by Eq. (16), and the Reynolds number Re, defined by Eq. (17), weed be specified to describe the flow.

Consider a pressure ratio of 50 . The flow was analyzed for several values of Re in order to display the effects of gas inertia. In Figs. 2, 3, and 4, results are presented for selected values of the dimensionless time $\tau$.

Figure 2 shows flow at zero Reynolds number, Darcy flow. For the largest time selected, $\tau$ equal to 3.0 , the pressure distribution is, for all practical purposes, fully developed. Calculated pressures at $\tau$ equal to 3.0 agree with those at $T$ equal to 2.5 to at least five digits. The flow can be considered to be in a steady state. For short times, $\tau$ less than about 0.38 , the effect of the distant boundary on the Darcy flow is negligible. The gas moves as if through a semi-ininite bed. The short time flow is similar. The sinilarity variable is $\theta$ and the early pressure history is very well described by the infinite pressure ratio curve of Fig. 1.

Figures 3 and 4 reveal how increasing Rey,olds number affects this behavior. Because of the added inertial resistance, the response is not as rapid. Neither of these flows is fully developed by $\tau$ equal to 3 . At 
least for the flow having a Reynolds number of 10 , the time scale $\mathrm{T}$, defined by Eq. (25), is more useful.

Changing scales, we will again describe the Re equal to $10, \mathrm{~N}$ equal to 50 flow. For selected values of the dimensionless time $T$, the pressure distribution is presented in Fig. 5. The full range of the flow response is observed within a range of $\mathrm{T}$ of order unity. In addition, and of greater importance, all flows possessing large Re and large $N$, and subject to conditions ( 45$)$, are virtually indistingushable from the flow presented in Fig. 5. In this high Re, high $\mathrm{N}$ range, the pressure distribution is nearly independent of Re and $N$ when presented as a function of $X$ and $T$.

The curve for $T$ equal to 3.0 does not corrospond to steady state. Only for much larger time is steady state achieved. This steady state pressure distribution is indeponden* of Reynolds number and the final distribution corresponds to the $\tau$ equal to 3.0 curve of Fig. 2 . The independence of the steady distribution from Reynolds number follows from Eqs. (18) and (19) with the temporal derivative set equal to zero. A non-Darcy flow resistance relation other than Forcheimer's relation would "ot necessarily yield this result.

For times prior to significant pressure change at $K$ equal to $l$, this high Re, high $N$ flow is nearly similar. It behaves as flow in a semi-infinite porous medium. The appropriate similarity variable is $\xi$, defined by Eq. (31) and describing the high Reynolds number flow governed by Eq. (32).

Results of calculations for very large Re and $n$ are shown in sinilar form in Fig. 6. N1l the curves of Fig. 4 as well as the short tine curves of Figs, 3 and 5 are well represented by this single curve. 


\section{CONCLUSICNS}

Non-Darcy fliw of an ideal gas has been investigated. Relations governing transient isothermal flow in one dimension were considered. Pressures resulting from a step chinge in pressure at the boundary of a semi-infinite bed were shown to depend on a single variable for high Reynolds number flow, A numerical technique was developed to describe transient flows in finite beds. Results have been obtained and presented slowing response characteristics of non-Darcy flows. 


\section{CAPTIONS FOR FIGURES}

Figure 1 Pressure distribution in an infinit: bed, low Reynolds number [19] Figure 2 Pressure distribution in a finite bed, Iow Reynolds number Figure 3 Pressure distribution in a finite bed, interuediate Reynolds number Figure 4 Pressure distribution in a finite bed, high Reynolds nubber Figure 5 Pressure distribution in a finite bed, high Reynolds number Figure 6 Pressure distribution in an infinite bed, high Reynolds number 


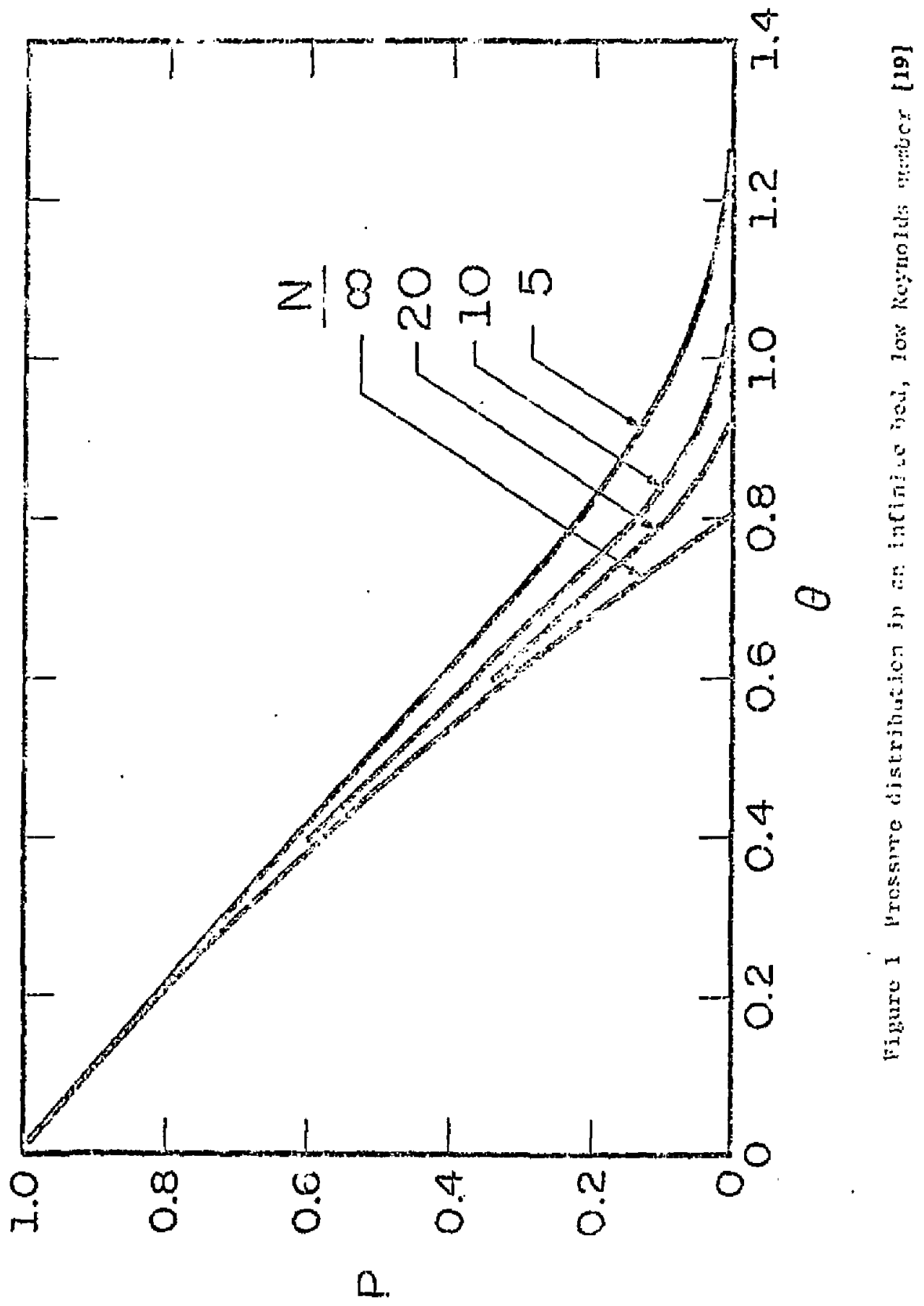




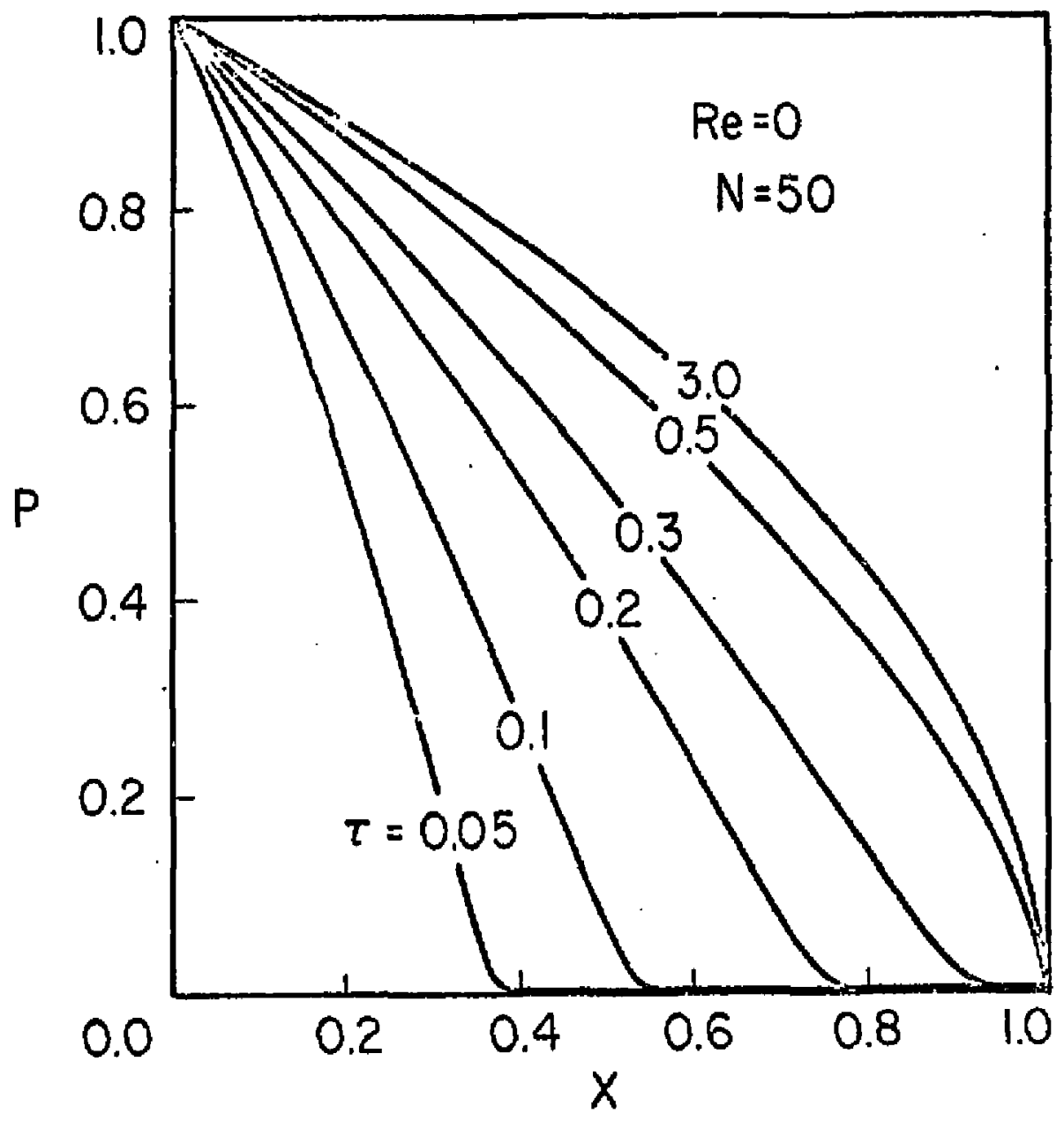

Figure 2 Pressure distribution in a finite bed, law Reynolds number. 


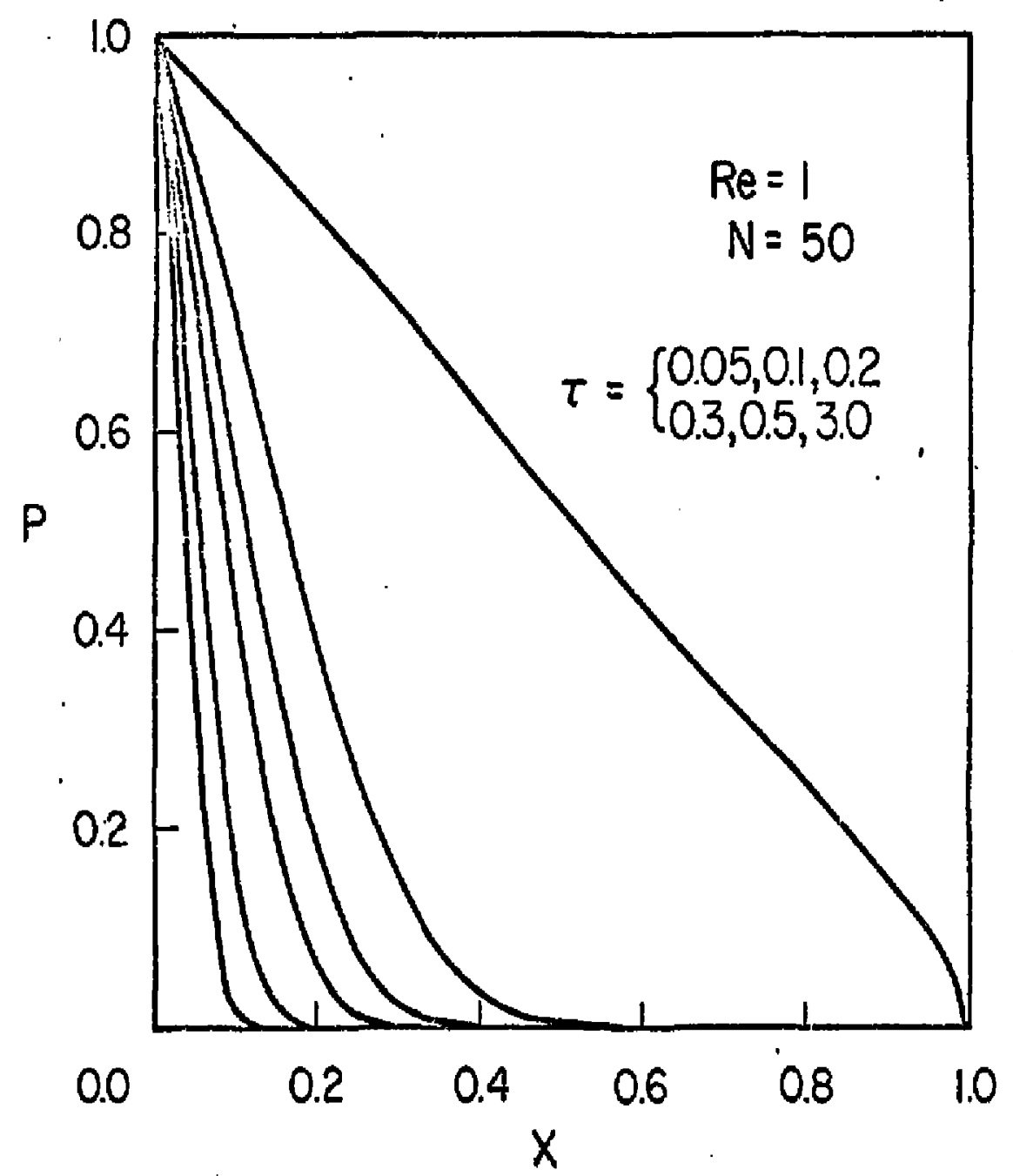

Figurc 5 Prossuro distribution in a tintle bed, inieractiate Reynolds muber 


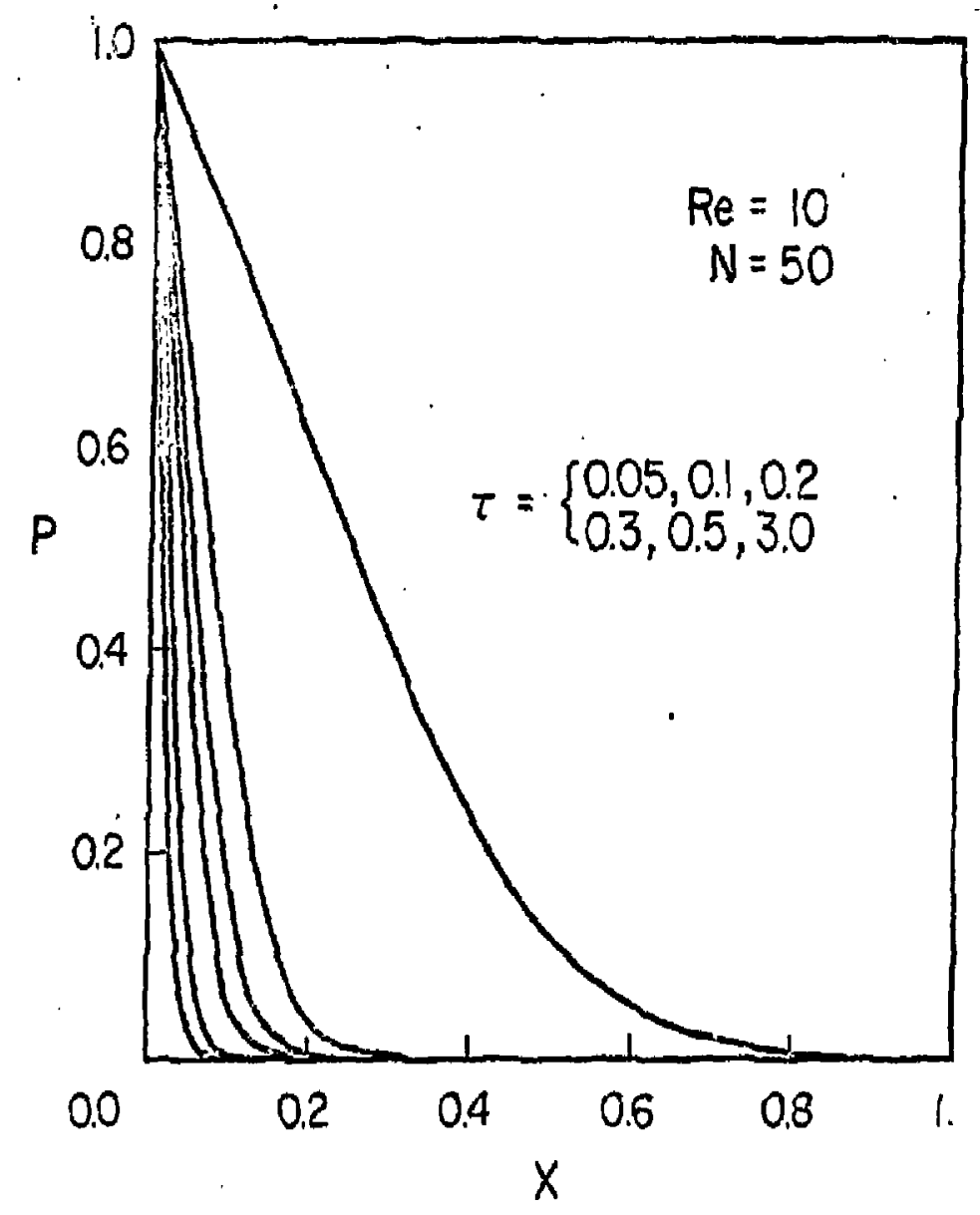

Figure A Pressure ristribution in a finite bed, high Reynolds nuiber 


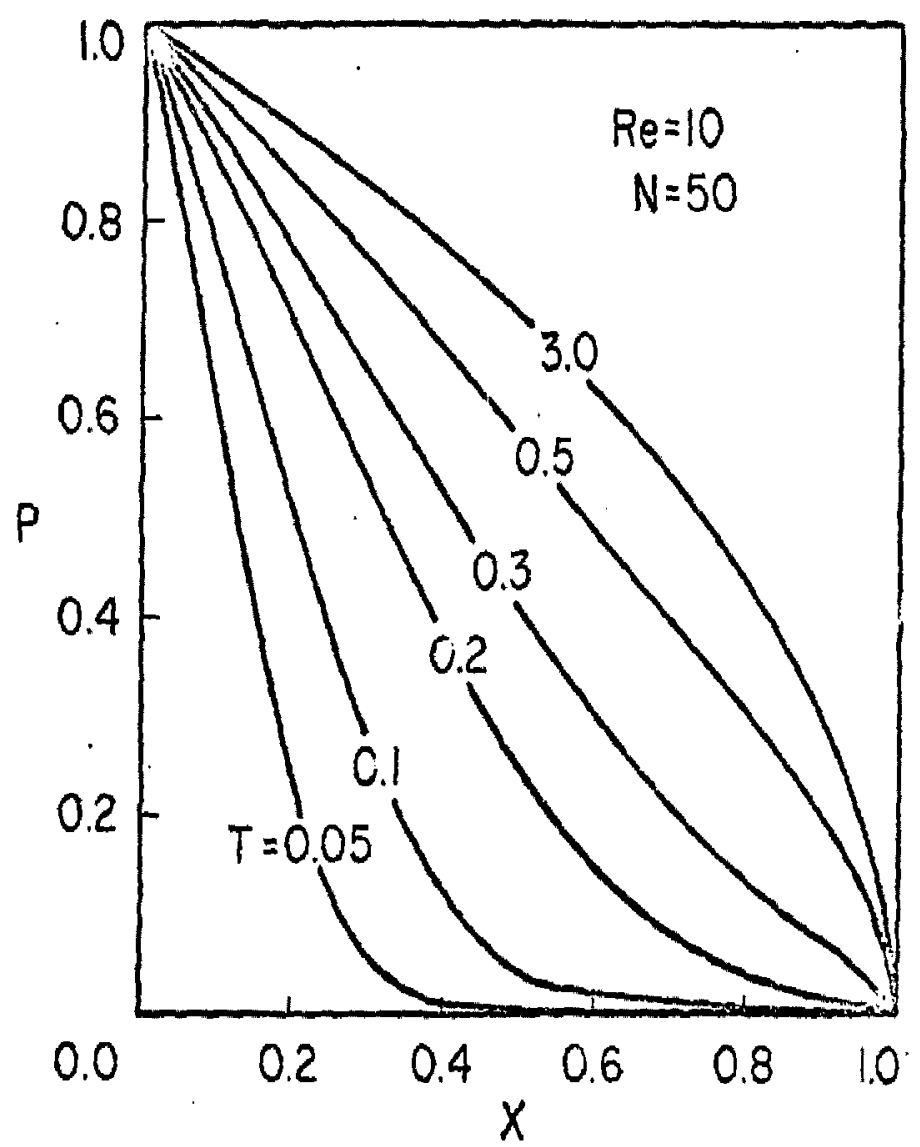

Figure 5 Pressure distribution in a finite bed, high Reynolds numbor 


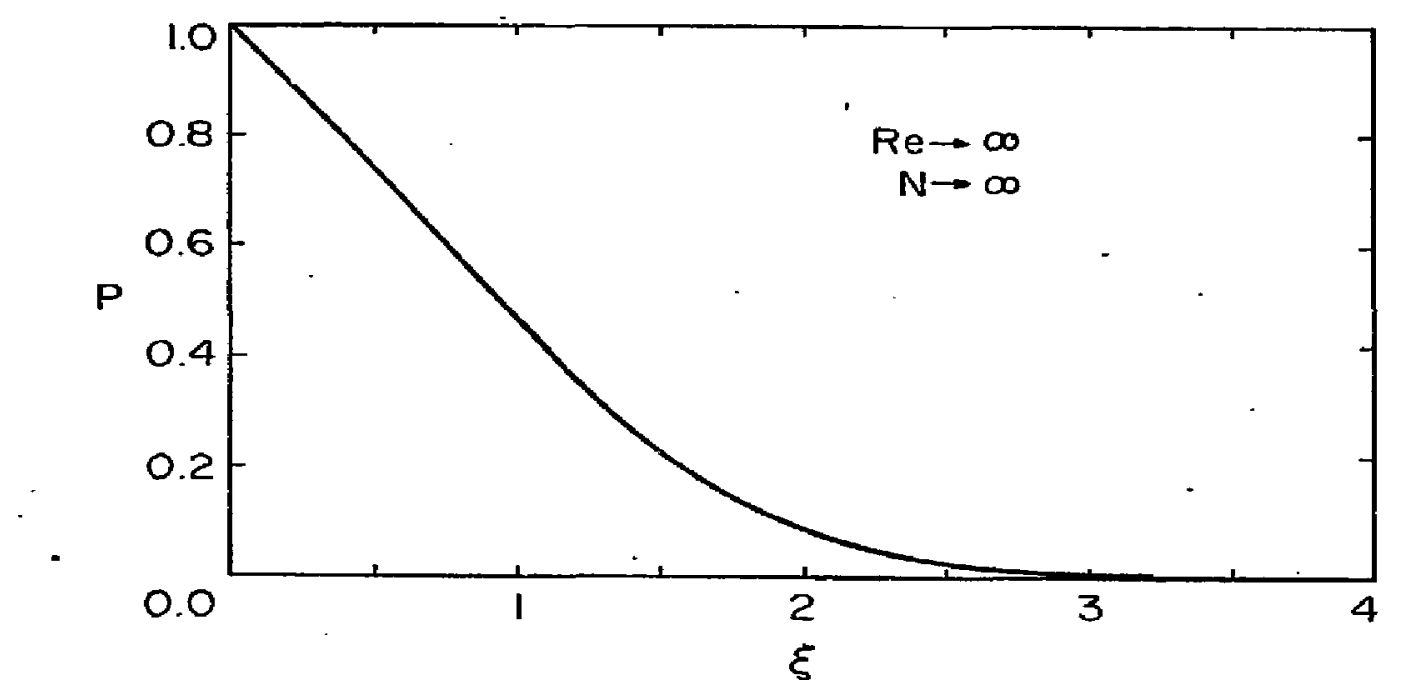

Figure 6 Pressure distribution in an infinite bed, high Reynolds number 


\section{REFERENCES}

1. Forcheincer, Ph.: "hisserbewegung durch Boden", Zeitschrift des Vereines Deutscher Ingenicure (1901) vo1. 45, 1781-1788.

2. Camman, P. C.: Flow of Gases through Porous Nedia, Academic Press, W.Y. (1956).

3. Happe1, J.: "Vistous Flow in Nultiparticle Systens: Slow Motion of Fuids felative to Beds of Spherical Particles", A.l.Ch.E.J. (1958) v01. 4, 197-201.

Happel, J.: "Fluid Wow in Multiparticle Systens", Trans. N.Y. Acad. Sci. (1958) vol. 20, 404-110.

4. Burke, S. P, and Plunner, W.B.: "Gas Flow through Packed Columns", Ind. ling. Chen. $(1928)$ vol, 20, 1196-1200.

5. Ergun, 5. and Orning, A. A.: "Fluid Flow through Randomly Packed Cojumats and Fluidized Beds", Ind. Eng. Chem. (1949) vol, 41, 1179-1184.

6. Green, l., Ir, and Duwez, P.: "Fluid Flow through Porous Metals", J. App1. Mech. $(145)$ ) vol. $18,39-45$.

7. Ergun, S.: "Fluid Flow through Packed Colum", Chem. Eng. Prog. (1952) vol. $48,89-94$.

8. Schnecholi, G.: "Experiences sur la linite de validite de la loi we larcy et l'apparition de la turbulence dans un écoulenent de filtratjon", La llouille bianche (2955) vol. 10, 141-149).

9. Ward, J,: "Turbulont Flow in Porous Modia", J, Mydraylics Biv. A.S.C.E. (1964) vol. 90, IIK5, 1-12.

10. Irmay, 5.: "On the Theoretical berivation of Darcy and lorchleimer Fonnulas", Trans., Moil (1958) Vol, 39, 702-707.

11. Bactllat, Y.: "Basic Transport Coefficients as Aqui fer Characteristics", I.A.S.II, Symp. Hodrology of lractured Rocks, nubrovnik (1965) vol, 1, $6.3-75$

12. Blick, E. f.,: "Capillasy-Orifice Nodel for lligh-Speed Flok through Porous Nedia", 1.GF.C. Process Dosign and bevelopment (1966) vol, 5, 90-94.

13. Polubarinova-kochiw, P. Ya.: Theory of Ground Water Movement, Princoton University Press, Princetan, N.J. (196\%).

14. Beavers, G. S. and Sparrous, E. M.: "Compressible Gas Flow through a Porous Matcrial", Int. J. lleat Mass Jimsfer (\$971) vol. 14, 18.55-1559. 
15. Masha, B. A., Beavers, G. S., and Sparrow, L. M.: "Experiments on the Resistance Law for Non-Darcy Compressible Gas Flows in Porous Medi:1", J. Fluids Engr., Trans., ASiE (1974) vol. 96, 353-357.

16. Swift, G. W. and Kiel, 0. G.: "rlie Prediction of Gas-liell Perforanace Including the Effact of Non-Darcy Flow", Trans., AlML (1962) vol. 225, 791-798.

17. Rowan, G. and Clegg, M. W.: "An Approximate Metliod for Non-Darcy Radial Gas Flow", [rans., AIME (1964) vol, 231, 96-114.

18. Dranchuk, P. M. and Chwyl, E.: "Transjent Gas Flow through Finite Linear Porous Modia", J. Can. Pet. Tech. (1969) vol. 8, 57-65.

19. Norrison, F, A., Jr.: "Transient Gas Flow in a Porous Column," I. \&l:.C. Fundamontals (1972) vol, 11, 191-197.

20. Norrison, F. A., Jr.: "Transient Mult jphase Multicomponent Flow in Porous Mcdia", lnt. J, Heat Mass Transfer (1973) vol. 16, 2333-2312.

21. Downing, R, S, and Morrison, F. A., Jr.: "Corvective and Dispersive Transjort in a Porous Nedium", to appear.

22. Dufort, L. C. and Frankel, S, P.: "Stability Considerations in the Nuacrical Treatment of Parabolic Differential Equations", Mlath. Tables Aids Camput. (1053) Wo1. 7, 135-152.

23. Saul'er, V. K.: "A Methor of Numerical Solution for the Diffusion Equation", Dok]. Akid. Naluk SSSR (1957) vol, 1]5, 1077-1079.

24. Larkin, B. K.: "Sone Stable Explinit Difference Approximitions to the Diffusion Equation", Math. of Comput, (1964) vol. 18, 196-202.

25. Barakat, II. Z. and Clark, J. A.: "On the Solution of the Diffusion Equations by Numerical Nothots", J. Heat Trinster, Trans. ASNE: (1966) vol. $88,421-427$.

26. Liu, S.-L.: "Stable Explicit Uifference Approximations to Parahalic Partial Differential liquations", A.I.Ch.E.J. (1969) vol, 15, 33.t-338.

27. Crank, J, and Nicolson, P.: "N Practical beili,d for Numerical thaluation of Solutions of Partial Differential Lquations of the lleat Contductior Type", Hroc. Camb. Phil. Ss.. (1947) vol. 43, 51/-67.

28. Bruce, G. HI., Peacetian, D. H., Rachford, H. Il, J J., and Ricc, J. D.: "Calculations of Unstoad'-State Gas Flow througll Porous Media", Trans., AlML (1955) vol, 198, 79-92, 